\title{
Convergence Analysis and Error Estimate for Distributed Optimal Control Problems Governed by Stokes Equations with Velocity-Constraint
}

\author{
Liang $\mathrm{Ge}^{1}$, Haifeng $\mathrm{Niu}^{2}$ and Jianwei Zhou ${ }^{3,4, *}$ \\ ${ }^{1}$ School of Mathematical Sciences, University of Jinan, Jinan 250022, Shandong, China \\ ${ }^{2}$ School of Mathematics and Information Science, Henan Polytechnic University, \\ Jiaozuo 454000, Henan, China \\ ${ }^{3}$ School of Mathematics and Statistics, Linyi University, Linyi 276005, Shandong, China \\ ${ }^{4}$ Division of Applied and Computational Mathematics, Beijing Computational Science \\ Research Center, Beijing 100193, China
}

Received 25 September 2020; Accepted (in revised version) 15 December 2020

\begin{abstract}
In this paper, spectral approximations for distributed optimal control problems governed by the Stokes equation are considered. And the constraint set on velocity is stated with $L^{2}$-norm. Optimality conditions of the continuous and discretized systems are deduced with the Karush-Kuhn-Tucker conditions and a Lagrange multiplier depending on the constraint. To solve the equivalent systems with high accuracy, Galerkin spectral approximations are employed to discretize the constrained optimal control systems. Meanwhile, we adopt a parameter $\lambda$ in the pressure approximation space, which also guarantees the inf-sup condition, and study a priori error estimates for the velocity and pressure. Specially, an efficient algorithm based on the Uzawa algorithm is proposed and its convergence results are investigated with rigorous analyses. Numerical experiments are performed to confirm the theoretical results.
\end{abstract}

AMS subject classifications: 49J20, 65N15, 65N35

Key words: Optimal control, spectral approximation, Stokes equation, convergence analysis.

\section{Introduction}

In recent years, there has been extensive research on theoretical and numerical results of optimal control problems governed by partial differential equations, and most of them are solved using finite element methods, see $[1,8,15,21,23-25]$ and the references cited therein. The authors in [20] employed finite element approximations to simulate the solutions of the optimality system, and derived optimal error estimates. In [19], the authors

*Corresponding author.

Emails: 1m_0202004@sina.com (L. Ge), hfniu@hpu.edu.cn (H. F. Niu), jwzhou@yahoo.com (J. W. Zhou) 
designed an efficient alternating direction method of multipliers for solving the optimal control problem problem by finite element methods. In [35], the authors derived optimal orders of convergence of state and adjoint state variables with mixed finite element methods. A penalized Neumann boundary control approach for optimal Dirichlet boundary control problems associated with steady-state Navier-Stokes equations was illustrated in [22]. The authors in [16] established the existence and first-order optimality condition of the optimal control with Navier-Stokes equations, and gave a convergence result on the augmented Lagrangian method for non-smooth cost functional. The authors in [38] employed finite element approximations to solve optimal control problems governed by time fractional diffusion equations. Furthermore, fully discrete schemes for time fractional optimal control problems were stated in [39].

Nowadays, both spectral methods and finite element methods are widely used for solving these problems. Further more, the literature about this topic is too huge to summarize. It is well-known that the spectral method provides a high accurate simulation if the solution is smooth enough [6]. Spectral methods for control-constrained optimal control problems were studied in $[10,11]$. Mixed spectral methods were proposed to solve the state-constrained optimal control problems in [36]. In engineering applications, optimal control systems have been used to describe the hydrodynamic models, and many research has been devoted to discussing optimal control problems in fluid dynamics. Stokes equations depict the motions of incompressible viscous fluid flow with low Reynolds numbers $[14,28]$ and the references cited therein. While the a posteriori error estimates were studied with the constraint on control [26], the a priori error estimates and a posteriori error estimates were investigated with the constraint on state [29,30]. Meanwhile, the a priori estimates and a posteriori error estimates of spectral approximations were stated in $[9,37]$. For lots of computational fluid dynamics, one often focuses on how to control the $L^{2}$-norm of velocity. In this paper, we adopt stationary Stokes equations to distributed optimal control problems and select the $L^{2}$-norm constraint on the velocity. To simplify the analysis and design of the system, we set the $L^{2}$-norm of velocity is not more than a given positive constant. Furthermore, we derive the equivalent optimality conditions and a priori error estimates in details. We also employ the Uzawa algorithm to design an efficient iterative algorithm. Meanwhile, we investigate the convergence of the algorithm with rigorous analyses.

The outline of this paper is organized as follows. In Section 2, we introduce the optimal control model and employ the Karush-Kuhn-Tucker conditions to investigate the first-order equivalent optimality conditions for the continuous systems. In Section 3, we give the Galerkin spectral approximations for the corresponding equivalent weak systems. In Section 4, we deduce the a priori error estimates for the spectral approximations with the help of two orthogonal projections and the Ladyzhenskaya-Babuška-Brezzi (LBB, or the inf-sup)-condition. Section 5 is devoted to designing an efficient algorithm to solve the coupled system. Meanwhile, the convergence of the given algorithm is proved. In Section 6, some numerical experiments are listed to validate the theoretical results. Finally, the conclusions are listed to summarize this paper. 


\section{Optimal control problem}

Throughout this paper, we adopt the standard notations of Sobolev spaces and the corresponding norms, semi-norms [2]. With $\Omega:=(-1,1)^{2} \subset \mathbb{R}^{2}$, we denote $\mathbf{v}$ a vector-valued function, and set $\mathbf{L}^{2}(\Omega)=\left(L^{2}(\Omega)\right)^{2}$ and $\mathbf{H}^{m}(\Omega)=\left(H^{m}(\Omega)\right)^{2}$ are vector-valued Sobolev spaces with norms $\|\cdot\|_{\mathbf{L}^{2}(\Omega)}=\|\cdot\|_{0, \Omega}$ and $\|\cdot\|_{\mathbf{H}^{m}(\Omega)}=\|\cdot\|_{m, \Omega}$, respectively. We use $c$ and $C$ to denote different constants in different formulas. We use

$$
\mathbf{U}=\left(L^{2}(\Omega)\right)^{2}, \quad \mathbf{V}=\left(H_{0}^{1}(\Omega)\right)^{2}, \quad W=\left\{w \in L^{2}(\Omega): \int_{\Omega} w=0\right\},
$$

to denote the control (i.e., body force) space, the state (i.e., velocity field) space and the pressure space, respectively.

\subsection{The model problem}

Two-dimensional incompressible Stokes equations read

$$
\begin{cases}-v \Delta \mathbf{u}+\nabla p=\mathbf{f} & \text { in } \Omega, \\ \nabla \cdot \mathbf{u}=0 & \text { in } \Omega, \\ \mathbf{u}=\mathbf{0} & \text { on } \partial \Omega,\end{cases}
$$

where $v>0$ is the kinematic viscosity, $\mathbf{u}$ is velocity, $p$ is pressure, the source term $\mathbf{f}$ is also named as the control variable. For a given $\mathbf{u}_{d} \in \mathbf{L}^{2}(\Omega)$, we define an objective functional

$$
\mathcal{J}(\mathbf{u}(\mathbf{f}), \mathbf{f})=\frac{1}{2} \int_{\Omega}\left|\mathbf{u}-\mathbf{u}_{d}\right|^{2}+\frac{\alpha}{2} \int_{\Omega}|\mathbf{f}|^{2} .
$$

Here, $\alpha$ is a regularization parameter, and for simplicity to describe the following analyses, we set $\alpha=1$. Note that $\mathcal{J}(\mathbf{u}, \mathbf{f})$ in (2.3) leads to the built-in coercivity, i.e., there exists a positive constant $\Theta$ such that

$$
(D \mathcal{J}(\mathbf{u}(\mathbf{f}), \mathbf{f})-D \mathcal{J}(\mathbf{v}(\mathbf{g}), \mathbf{g}), \mathbf{f}-\mathbf{g}) \geq \Theta\|\mathbf{f}-\mathbf{g}\|_{0, \Omega}^{2},
$$

where $D \mathcal{J}(\mathbf{u}(\mathbf{f}), \mathbf{f}) \cdot \mathbf{v}$ denotes the Gâteaux derivative of $\mathcal{J}(\mathbf{u}(\mathbf{f}), \mathbf{f})$ following any direction v. An interesting optimal control problem and its equivalent optimality conditions were stated step by step. We are interested in the following state-constrained optimal control problem

$$
\min _{\mathbf{u} \in K} \mathcal{J}(\mathbf{u}, \mathbf{f})
$$

subjected to (2.1), and the constraint $K$ on the velocity is defined as

$$
K=\left\{\mathbf{v} \in \mathbf{V}:\|\mathbf{v}\|_{0, \Omega} \leq \gamma, \gamma>0\right\} .
$$


To deduce a weak formulation of (2.4), we define

$$
\begin{aligned}
& (\mathbf{x}, \mathbf{v})=\int_{\Omega} \mathbf{x} \cdot \mathbf{v}, \quad a(\mathbf{w}, \mathbf{z})=v(\nabla \mathbf{w}, \nabla \mathbf{z}), \\
& b(\mathbf{z}, q)=-(\nabla \cdot \mathbf{z}, q), \quad \forall \mathbf{x}, \mathbf{v} \in \mathbf{U}, \quad \forall \mathbf{z}, \mathbf{w} \in \mathbf{V}, \quad \forall q \in W .
\end{aligned}
$$

It is clear that the bi-linear form $a(\cdot, \cdot)$ is continuous and elliptic in $\mathbf{V}$. Additionally, it has been discussed in [14] that, $b(\cdot, \cdot)$ satisfies the LBB-condition: for $\forall \mathbf{v} \in \mathbf{V}$, there exists a constant $C_{\Omega}>0$ such that

$$
C_{\Omega}\|q\|_{0, \Omega} \leq \sup _{\mathbf{z} \in \mathbf{V}} \frac{b(\mathbf{z}, q)}{|\mathbf{z}|_{1, \Omega}}, \quad \forall q \in W .
$$

In view of (2.6), the weak formulation of the optimal control problem (2.4) reads:

$$
\begin{aligned}
& \min _{\mathbf{u} \in K} \mathcal{J}(\mathbf{u}(\mathbf{f}), \mathbf{f}) \\
& \text { s.t. } \begin{cases}a(\mathbf{u}, \mathbf{w})+b(\mathbf{w}, p)=(\mathbf{f}, \mathbf{w}), & \forall \mathbf{w} \in \mathbf{V}, \\
b(\mathbf{u}, q)=0, & \forall q \in W .\end{cases}
\end{aligned}
$$

The existence and uniqueness of the solution for (2.8) can be obtained by using standard approaches as in [24].

\subsection{Optimality condition}

We employ the Karush-Kuhn-Tucker conditions [13] to deduce the first-order optimality conditions of (2.8). If $\mathbf{f}$ is the solution of (2.8), there exists a constant $t$, which is named as Lagrange multiplier, such that

$$
\begin{aligned}
& t \geq 0, \quad t F(\mathbf{f})=0, \\
& \frac{\partial \mathscr{L}(\mathbf{f}, t)}{\partial \mathbf{f}} \cdot \mathbf{v}=0, \quad \forall \mathbf{v} \in \mathbf{U},
\end{aligned}
$$

where the Lagrange functional $\mathscr{L}(\mathbf{f}, t): \mathbf{U} \times \mathbb{R} \mapsto \mathbb{R}$ is defined as

$$
\mathscr{L}(\mathbf{f}, t):=\mathcal{J}(\mathbf{u}(\mathbf{f}), \mathbf{f})+t F(\mathbf{f}), \quad F(\mathbf{f}):=\|\mathbf{u}(\mathbf{f})\|_{0, \Omega}-\gamma .
$$

Here we give the first-order equivalent optimality conditions for the problem (2.8).

Theorem 2.1. The triplet $(\mathbf{u}, p, \mathbf{f}) \in \mathbf{V} \times W \times \mathbf{U}$ is the solution of (2.8) if and only if there exists a unique triplet $\left(\mathbf{u}^{*}, p^{*}, t\right) \in \mathbf{V} \times W \times \mathbb{R}$ satisfies

$$
\begin{cases}\text { (a) } a(\mathbf{u}, \mathbf{w})+b(\mathbf{w}, p)=(\mathbf{f}, \mathbf{w}), & \forall \mathbf{w} \in \mathbf{V}, \\ \text { (b) } b(\mathbf{u}, q)=0, & \forall q \in W, \\ \text { (c) } a\left(\mathbf{u}^{*}, \mathbf{w}\right)+b\left(\mathbf{w}, p^{*}\right)=\left(\left(1+\frac{t}{\gamma}\right) \mathbf{u}-\mathbf{u}_{d}, \mathbf{w}\right), & \forall \mathbf{w} \in \mathbf{V}, \\ \text { (d) } b\left(\mathbf{u}^{*}, q\right)=0, & \forall q \in W, \\ (e) t(\mathbf{u}, \mathbf{w}-\mathbf{u}) \leq 0, & \forall \mathbf{w} \in K, \\ \text { (f) } \mathbf{u}^{*}+\mathbf{f}=\mathbf{0} . & \end{cases}
$$


And the solution is unique. Furthermore,

$$
t= \begin{cases}0, & \text { if }\|\mathbf{u}\|_{0, \Omega}<\gamma, \\ c \geq 0, & \text { if }\|\mathbf{u}\|_{0, \Omega}=\gamma .\end{cases}
$$

Proof. Combining (2.6) with (2.1), we readily deduce that (2.11)-(a) and (2.11)-(b) hold. Following (2.9a), one obtains that there exists a constant $t$ satisfying

$$
t\left(\|\mathbf{u}\|_{0, \Omega}-\gamma\right)=0 .
$$

We study the properties of $t$ with two cases. Firstly, if $\|\mathbf{u}\|_{0, \Omega}<\gamma$, there holds $t=0$, i.e., (2.11)-(e) naturally holds. Secondly, if $\|\mathbf{u}\|_{0, \Omega}=\gamma$, for $\forall \mathbf{w} \in K$, then

$$
t(\mathbf{u}, \mathbf{w}-\mathbf{u})=t(\mathbf{u}, \mathbf{w})-t \gamma^{2} \leq t \gamma\left(\|\mathbf{w}\|_{0, \Omega}-\gamma\right) \leq 0 .
$$

According to the above two cases, we declare that (2.11)-(e) and (2.12) hold. Furthermore, we calculate the Gâteaux derivative of $\mathscr{L}(\mathbf{f}, t)$ in any direction $\mathbf{v}$ as

$$
\begin{aligned}
\frac{\partial \mathscr{L}(\mathbf{f}, t)}{\partial \mathbf{f}} \cdot \mathbf{v} & =\left(\mathbf{u}-\mathbf{u}_{d}, D \mathbf{u}(\mathbf{f}) \cdot \mathbf{v}\right)+(\mathbf{f}, D \mathbf{f}(\mathbf{f}) \cdot \mathbf{v})+\frac{t}{\gamma}(\mathbf{u}, D \mathbf{u}(\mathbf{f}) \cdot \mathbf{v}) \\
& =\left(\left(\mathbf{u}-\mathbf{u}_{d}+\frac{t}{\gamma} \mathbf{u}\right), D \mathbf{u}(\mathbf{f}) \cdot \mathbf{v}\right)+(\mathbf{f}, \mathbf{v})
\end{aligned}
$$

where $D \mathbf{u}(\mathbf{f}) \cdot \mathbf{v}$ denotes the Gateaux derivative of $\mathbf{u}(\mathbf{f})$ in the direction $\mathbf{v}$. Now we are at the point to investigate the properties of $\left(\mathbf{u}-\mathbf{u}_{d}+\frac{t}{\gamma} \mathbf{u}, D \mathbf{u}(\mathbf{f}) \cdot \mathbf{v}\right)$. By (2.11)-(a) and (2.11)(b), we readily get

$$
\begin{aligned}
& a(D \mathbf{u}(\mathbf{f}) \cdot \mathbf{v}, \mathbf{w})+b(\mathbf{w}, D p(\mathbf{f}) \cdot \mathbf{v})=(\mathbf{v}, \mathbf{w}), \\
& b(D \mathbf{u}(\mathbf{f}) \cdot \mathbf{v}, q)=0 .
\end{aligned}
$$

Taking $\mathbf{v}=\mathbf{u}-\mathbf{u}_{d}+\frac{t}{\gamma} \mathbf{u}$ and $\mathbf{w}=D \mathbf{u}(\mathbf{f}) \cdot \mathbf{v}$, there holds

$$
\begin{aligned}
& \left(\mathbf{u}-\mathbf{u}_{d}+\frac{t}{\gamma} \mathbf{u}, D \mathbf{u}(\mathbf{f}) \cdot \mathbf{v}\right) \\
= & a\left(D \mathbf{u}(\mathbf{f}) \cdot\left(\mathbf{u}-\mathbf{u}_{d}+\frac{t}{\gamma} \mathbf{u}\right), D \mathbf{u}(\mathbf{f}) \cdot \mathbf{v}\right)+b\left(D \mathbf{u}(\mathbf{f}) \cdot \mathbf{v}, D p(\mathbf{f}) \cdot\left(\mathbf{u}-\mathbf{u}_{d}+\frac{t}{\gamma} \mathbf{u}\right)\right) .
\end{aligned}
$$

Meanwhile, setting

$$
\mathbf{w}=D \mathbf{u}(\mathbf{f}) \cdot\left(\mathbf{u}-\mathbf{u}_{d}+\frac{t}{\gamma} \mathbf{u}\right)
$$

there holds

$$
\begin{aligned}
& \left(\mathbf{v}, D \mathbf{u}(\mathbf{f}) \cdot\left(\mathbf{u}-\mathbf{u}_{d}+\frac{t}{\gamma} \mathbf{u}\right)\right) \\
= & a\left(D \mathbf{u}(\mathbf{f}) \cdot \mathbf{v}, D \mathbf{u}(\mathbf{f}) \cdot\left(\mathbf{u}-\mathbf{u}_{d}+\frac{t}{\gamma} \mathbf{u}\right)\right)+b\left(D \mathbf{u}(\mathbf{f}) \cdot\left(\mathbf{u}-\mathbf{u}_{d}+\frac{t}{\gamma} \mathbf{u}\right), D p(\mathbf{f}) \cdot \mathbf{v}\right) .
\end{aligned}
$$


With (2.13) and (2.6), direct calculations of the Gâteaux derivative show that, for any $\mathbf{v} \in \mathbf{U}$, there holds

$$
\frac{\partial \mathscr{L}(\mathbf{f}, t)}{\partial \mathbf{f}} \cdot \mathbf{v}=\left(D \mathbf{u}(\mathbf{f}) \cdot\left(\mathbf{u}-\mathbf{u}_{d}+\frac{t}{\gamma} \mathbf{u}\right), \mathbf{v}\right)+(\mathbf{f}, \mathbf{v})=\left(\mathbf{u}^{*}+\mathbf{f}, \mathbf{v}\right),
$$

where

$$
\mathbf{u}^{*}=D \mathbf{u}(\mathbf{f}) \cdot\left(\mathbf{u}-\mathbf{u}_{d}+\frac{t}{\gamma} \mathbf{u}\right)
$$

By $(2.9 b)$, one can also directly check the following identity

$$
\mathbf{u}^{*}+\mathbf{f}=0 \text {, }
$$

which is consistent with (2.11)-(f). Taking

$$
\mathbf{v}=\mathbf{u}-\mathbf{u}_{d}+\frac{t}{\gamma} \mathbf{u} \in \mathbf{U}
$$

in (2.14), we have

$$
a\left(\mathbf{u}^{*}, \mathbf{w}\right)+b\left(\mathbf{w}, p^{*}\right)=\left(\mathbf{u}-\mathbf{u}_{d}+\frac{t}{\gamma} \mathbf{u}, \mathbf{w}\right)
$$

where

$$
p^{*}=D p(\mathbf{f}) \cdot\left(\mathbf{u}-\mathbf{u}_{d}+\frac{t}{\gamma} \mathbf{u}\right)
$$

i.e., (2.11)-(c) holds. Employing (2.11)-(b), and setting

$$
\mathbf{v}=\mathbf{u}-\mathbf{u}_{d}+\frac{t}{\gamma} \mathbf{u} \in \mathbf{U}
$$

one readily deduces that

$$
b\left(\mathbf{u}^{*}, q\right)=0,
$$

where $\mathbf{u}^{*}$ is defined as in (2.15), this proves (2.11)-(d).

Finally, we turn to prove the uniqueness of the solution of (2.11). Thereby we assume that there exist two solutions $\left(\mathbf{u}_{1}, p_{1}, \mathbf{f}_{1}, \mathbf{u}_{1}^{*}, p_{1}^{*}, t_{1}\right)$ and $\left(\mathbf{u}_{2}, p_{2}, \mathbf{f}_{2}, \mathbf{u}_{2}^{*}, p_{2}^{*}, t_{2}\right)$. With (2.11)-(a) and (2.11)-(c), we have

$$
\begin{aligned}
& a\left(\mathbf{u}_{1}-\mathbf{u}_{2}, \mathbf{w}\right)+b\left(\mathbf{w}, p_{1}-p_{2}\right)=\left(\mathbf{f}_{1}-\mathbf{f}_{2}, \mathbf{w}\right), \\
& a\left(\mathbf{u}_{1}^{*}-\mathbf{u}_{2}^{*}, \mathbf{w}\right)+b\left(\mathbf{w}, p_{1}^{*}-p_{2}^{*}\right)=\frac{1}{\gamma}\left(t_{1} \mathbf{u}_{1}-t_{2} \mathbf{u}_{2}, \mathbf{w}\right)+\left(\mathbf{u}_{1}-\mathbf{u}_{2}, \mathbf{w}\right) .
\end{aligned}
$$


In the light of setting $\mathbf{w}=\mathbf{u}_{1}^{*}-\mathbf{u}_{2}^{*}$ and $\mathbf{w}=\mathbf{u}_{1}-\mathbf{u}_{2}$ in (2.18a) and (2.18b), respectively, we obtain

$$
\begin{aligned}
& a\left(\mathbf{u}_{1}-\mathbf{u}_{2}, \mathbf{u}_{1}^{*}-\mathbf{u}_{2}^{*}\right)+b\left(\mathbf{u}_{1}^{*}-\mathbf{u}_{2}^{*}, p_{1}-p_{2}\right)=\left(\mathbf{f}_{1}-\mathbf{f}_{2}, \mathbf{u}_{1}^{*}-\mathbf{u}_{2}^{*}\right)=-\left\|\mathbf{f}_{1}-\mathbf{f}_{2}\right\|_{0, \Omega}^{2} \\
& a\left(\mathbf{u}_{1}^{*}-\mathbf{u}_{2}^{*}, \mathbf{u}_{1}-\mathbf{u}_{2}\right)+b\left(\mathbf{u}_{1}-\mathbf{u}_{2}, p_{1}^{*}-p_{2}^{*}\right)=\frac{1}{\gamma}\left(t_{1} \mathbf{u}_{1}-t_{2} \mathbf{u}_{2}, \mathbf{u}_{1}-\mathbf{u}_{2}\right)+\left\|\mathbf{u}_{1}-\mathbf{u}_{2}\right\|_{0, \Omega}^{2},
\end{aligned}
$$

where we used (2.11)-(f). Since $p_{1}-p_{2} \in W, p_{1}^{*}-p_{2}^{*} \in W$, combining (2.11)-(b), (2.11)-(d) with $t \geq 0, \gamma>0$, one directly gets

$$
\frac{1}{\gamma}\left(t_{1} \mathbf{u}_{1}-t_{2} \mathbf{u}_{2}, \mathbf{u}_{1}-\mathbf{u}_{2}\right)+\left\|\mathbf{f}_{1}-\mathbf{f}_{2}\right\|_{0, \Omega}^{2}+\left\|\mathbf{u}_{1}-\mathbf{u}_{2}\right\|_{0, \Omega}^{2}=0 .
$$

With the help of (2.11)-(e), we declare that

$$
\frac{1}{\gamma}\left(t_{1} \mathbf{u}_{1}-t_{2} \mathbf{u}_{2}, \mathbf{u}_{1}-\mathbf{u}_{2}\right) \geq 0
$$

In view of (2.19) and (2.20), it is a direct conclusion that $\mathbf{f}_{1}=\mathbf{f}_{2}$ and $\mathbf{u}_{1}=\mathbf{u}_{2}$. Consequently, with the help of (2.11)-(b), (2.11)-(f) and (2.18a), we obtain that $\mathbf{u}_{1}^{*}=\mathbf{u}_{2}^{*}$ and $p_{1}=p_{2}$, respectively.

Similarly, combining the above equalities with (2.18b), for any $\mathbf{w} \in \mathbf{V}$, we get that

$$
\frac{1}{\gamma}\left(\left(t_{1}-t_{2}\right) \mathbf{u}_{1}, \mathbf{w}\right)=0
$$

One can readily declare that there holds

$$
t_{1}=t_{2} \quad \text { a.e. in } \Omega \text {. }
$$

Hence, we complete the proof.

Remark 2.1. In the above proof, we used the fact that

$$
\frac{t}{\sqrt{\int_{\Omega}|\mathbf{u}|^{2}}}=\frac{t}{\gamma} .
$$

Indeed, if $\|\mathbf{u}\|_{0, \Omega}<\gamma$, there holds $t=0$. Otherwise, $\|\mathbf{u}\|_{0, \Omega}=\gamma$ is trivial.

Remark 2.2. By the above analyses, regularities of the solutions can be enhanced step by step. 


\section{Spectral-Galerkin approximation}

In this section, we investigate the Galerkin spectral approximations for the model problems. Let $\mathcal{P}_{N}$ be the space of polynomials $p(x, y)$ whose degree in each direction is not more than $N$. Then we set $P_{N}=\left\{v \in \mathcal{P}_{N}:\left.v\right|_{\partial \Omega}=0\right\}$. We define

$$
\mathbf{U}_{N}=\left(P_{N}, P_{N}\right), \quad \mathbf{V}_{N}=\mathbf{U}_{N} \cap \mathbf{V}, \quad W_{\bar{N}}=P_{[\lambda N]} \cap W,
$$

where $\lambda \in(0,1)$ is an artificial parameter. $\mathbf{V}_{N}$ denotes the approximation space for the velocity $\mathbf{u}$ and its adjoint $\mathbf{u}^{*}, W_{\bar{N}}$ for the pressure $p$ and its adjoint $p^{*}$, and $\mathbf{U}_{N}$ for the control f.

We define

$$
\phi_{i}(x)=\frac{L_{i}(x)-L_{i+2}(x)}{\sqrt{4 i+6}},
$$

where $L_{i}(x)$ denotes the orthogonal Legendre polynomial on $[-1,1]$. Then, we have

$$
\mathbf{V}_{N}=\left\{\left(\phi_{i}(x) \phi_{j}(y), \phi_{k}(x) \phi_{l}(y)\right), i, j, k, l=0,1, \cdots, N-2\right\} .
$$

Then the Galerkin spectral approximations of the optimal control problem (2.8) is: find $\left(\mathbf{u}_{N}, \mathbf{f}_{N}, p_{N}\right) \in \mathbf{V}_{N} \times \mathbf{U}_{N} \times W_{\bar{N}}$ such that

$$
\begin{aligned}
& \min _{\mathbf{u}_{N} \in \mathbf{K} \cap \mathbf{U}_{N}} \mathcal{J}\left(\mathbf{u}_{N}, \mathbf{f}_{N}\right) \\
& \begin{cases}a\left(\mathbf{u}_{N}, \mathbf{v}_{N}\right)+b\left(\mathbf{v}_{N}, p_{N}\right)=\left(\mathbf{f}_{N}, \mathbf{v}_{N}\right), & \forall \mathbf{v}_{N} \in \mathbf{V}_{N}, \\
b\left(\mathbf{u}_{N}, q_{N}\right)=0, & \forall q_{N} \in W_{\bar{N}} .\end{cases}
\end{aligned}
$$

The existence and uniqueness of the solution for (3.2) can be obtained similar as that for the continuous problem (2.8), using standard approaches as in [14,17,24].

We recall the following important result [5, pp. 399, Proposition 3.1]:

Lemma 3.1 ([5]). For any real number $\lambda \in(0,1)$ and $N \geq 2 /(1-\lambda)$, there exists a positive constant $\beta$ independent of $N$ such that

$$
\sup _{\mathbf{v}_{N} \in \mathbf{V}_{N}} \frac{\left(q_{N}, \nabla \cdot \mathbf{v}_{N}\right)}{\left\|\mathbf{v}_{N}\right\|_{1, \Omega}} \geq \beta\left\|q_{N}\right\|_{L^{2}(\Omega)}, \quad \forall q_{N} \in W_{\bar{N}}
$$

The constant $\beta$ is the inf-sup constant (for more details of this constant, we refer the readers to [5]).

Remark 3.1. As that in [5, Section 3], for any given $\lambda \in(0,1)$, the state equation in (3.2) is well-posed with

$$
W_{N}^{(\lambda)}=\left\{q \in P_{[\lambda N]}: \int_{\Omega} q \mathrm{~d} x=0\right\},
$$


and $\beta$ in (3.3) is independent of $N$ but dependent on $\lambda$ with $\beta \rightarrow 0$ as $\lambda \rightarrow 1^{-}$.

Furthermore, if

$$
W_{\bar{N}}=P_{[\lambda N]-1} \cap W,
$$

inequality (3.3) is still valid and the constraint $N \geq 2 /(1-\lambda)$ vanishes. More details, kindly please refer to [5].

By using the same lines of arguments as in the proof of Theorem 2.1, we have the following result for the optimality conditions of (3.2).

Theorem 3.1. The triplet $\left(\mathbf{u}_{N}, p_{N}, \mathbf{f}_{N}\right) \in \mathbf{V}_{N} \times W_{\bar{N}} \times \mathbf{U}_{N}$ is the solution of the problem (3.2) if and only if there exists a unique triplet $\left(\mathbf{u}_{N}^{*}, p_{N}^{*}, t_{N}\right) \in \mathbf{V}_{N} \times W_{\bar{N}} \times \mathbb{R}$ such that

$$
\begin{cases}\text { (a) } a\left(\mathbf{u}_{N}, \mathbf{w}_{N}\right)+b\left(\mathbf{w}_{N}, p_{N}\right)=\left(\mathbf{f}_{N}, \mathbf{w}_{N}\right), & \forall \mathbf{w}_{N} \in \mathbf{V}_{N}, \\ \text { (b) } b\left(\mathbf{u}_{N}, q_{N}\right)=0, & \forall q_{N} \in W_{\bar{N}}, \\ \text { (c) } a\left(\mathbf{u}_{N}^{*}, \mathbf{w}_{N}\right)+b\left(\mathbf{w}_{N}, p_{N}^{*}\right)=\left(\left(1+\frac{t_{N}}{\gamma}\right) \mathbf{u}_{N}-\mathbf{u}_{d}, \mathbf{w}_{N}\right), & \forall \mathbf{w}_{N} \in \mathbf{V}_{N}, \\ \text { (d) } b\left(\mathbf{u}_{N}^{*}, q_{N}\right)=0, & \forall q_{N} \in W_{\bar{N}}, \\ \text { (e) } t_{N}\left(\mathbf{u}_{N}, \mathbf{w}_{N}-\mathbf{u}_{N}\right) \leq 0, & \forall \mathbf{w}_{N} \in K, \\ \text { (f) } \mathbf{u}_{N}^{*}+\mathbf{f}_{N}=\mathbf{0} . & \end{cases}
$$

And the constant $t_{N}$ satisfies

$$
t_{N}= \begin{cases}0, & \text { if }\left\|\mathbf{u}_{N}\right\|_{0, \Omega}<\gamma, \\ c \geq 0, & \text { if }\left\|\mathbf{u}_{N}\right\|_{0, \Omega}=\gamma .\end{cases}
$$

\section{A priori error estimate}

In this section, we derive a priori error estimates for the Galerkin spectral approximation. We recall two projection operators. For $v \in L^{2}(\Omega)$, we define the $L^{2}$-projection operator $\mathbb{P}_{N}: L^{2}(\Omega) \mapsto \mathcal{P}_{N}$ by

$$
\left(\mathbb{P}_{N} v-v, w_{N}\right)=0, \quad \forall w_{N} \in \mathcal{P}_{N} .
$$

Similarly, for $\forall v \in H_{0}^{1}(\Omega)$, we define $\mathbb{P}_{1, N}^{0}: H_{0}^{1}(\Omega) \mapsto P_{N}$ by

$$
a\left(\mathbb{P}_{1, N}^{0} v-v, w_{N}\right)=0, \quad \forall w_{N} \in P_{N} .
$$

With the tensor product, these projections can be expanded to vector space $\mathbf{U}_{N}$ and $\mathbf{V}_{N}$ on each direction, respectively.

Lemma 4.1 ([6]). For all $v \in H^{m}(\Omega)(m \geq 1)$, there holds

$$
\left\|v-\mathbb{P}_{N} v\right\|_{l, \Omega} \leq c N^{\sigma(l)-m}\|v\|_{m, \Omega}, \quad 0 \leq l \leq m,
$$


where

$$
\sigma(l)= \begin{cases}2 l-\frac{1}{2}, & \text { if } l \geq 1, \\ \frac{3}{2} l, & \text { if } 0 \leq l \leq 1 .\end{cases}
$$

For all $v \in H_{0}^{1}(\Omega) \cap H^{m}(\Omega)(m \geq 1)$, there holds

$$
\left\|v-\mathbb{P}_{1, N}^{0} v\right\|_{l, \Omega} \leq c N^{l-m}\|v\|_{m, \Omega}, \quad l=0,1 .
$$

With a litter abuse of notation, the same symbols are used to denote the corresponding operators on vector and scalar spaces.

One directly proves that there exists a positive constant $C$ such that [29]

$$
\left\|\mathbf{f}_{N}\right\|_{0, \Omega}+\left\|\mathbf{u}_{N}\right\|_{1, \Omega}+\left\|p_{N}\right\|_{0, \Omega}+\left\|\mathbf{u}_{N}^{*}\right\|_{1, \Omega}+\left\|p_{N}^{*}\right\|_{0, \Omega}+\left|t_{N}\right| \leq C .
$$

We assume the solutions of the optimality conditions satisfy $\mathbf{u}, \mathbf{u}^{*} \in H^{m}(\Omega)$, and $p, p^{*} \in$ $H^{m-1}(\Omega)(m \geq 1)$. And in order to investigate a priori error estimates, we introduce an auxiliary system

$$
\begin{cases}a\left(\mathbf{u}_{N}(\mathbf{f}), \mathbf{v}_{N}\right)+b\left(\mathbf{v}_{N}, p_{N}(\mathbf{f})\right)=\left(\mathbf{f}, \mathbf{v}_{N}\right), & \forall \mathbf{v}_{N} \in \mathbf{V}_{N}, \\ b\left(\mathbf{u}_{N}(\mathbf{f}), w_{N}\right)=0, & \forall w_{N} \in W_{\bar{N}}, \\ a\left(\mathbf{u}_{N}^{*}(\mathbf{f}), \mathbf{v}_{N}\right)+b\left(\mathbf{v}_{N}, p_{N}^{*}(\mathbf{f})\right)=\left(\left(1+\frac{t}{\gamma}\right) \mathbf{u}_{N}(\mathbf{f})-\mathbf{u}_{d}, \mathbf{v}_{N}\right) & \forall \mathbf{v}_{N} \in \mathbf{V}_{N}, \\ b\left(\mathbf{u}_{N}^{*}(\mathbf{f}), w_{N}\right)=0, & \forall w_{N} \in W_{\bar{N}} .\end{cases}
$$

Lemma 4.2. Let $\left(\mathbf{u}_{N}(\mathbf{f}), p_{N}(\mathbf{f}), \mathbf{u}_{N}^{*}(\mathbf{f}), p_{N}^{*}(\mathbf{f}), t, \mathbf{f}\right)$ and $\left(\mathbf{u}_{N}, p_{N}, \mathbf{u}_{N}^{*}, p_{N}^{*}, t_{N}, \mathbf{f}_{N}\right)$ be the solutions of (4.6) and (3.4), respectively. Then there holds

$$
\left\|\mathbf{u}_{N}(\mathbf{f})-\mathbf{u}_{N}\right\|_{1, \Omega}+\left\|p_{N}(\mathbf{f})-p_{N}\right\|_{0, \Omega} \leq C\left\{\left(1+N^{-1}\right)\left\|\mathbf{f}-\mathbb{P}_{N} \mathbf{f}\right\|_{0, \Omega}+2\left\|\mathbb{P}_{N} \mathbf{f}-\mathbf{f}_{N}\right\|_{0, \Omega}\right\} .
$$

Proof. Subtracting (3.4) from (4.6), we obtain

$$
\begin{cases}a\left(\mathbf{u}_{N}(\mathbf{f})-\mathbf{u}_{N}, \mathbf{v}_{N}\right)+b\left(\mathbf{v}_{N}, p_{N}(\mathbf{f})-p_{N}\right)=\left(\mathbf{f}-\mathbf{f}_{N}, \mathbf{v}_{N}\right), & \forall \mathbf{v}_{N} \in \mathbf{V}_{N} \\ b\left(\mathbf{u}_{N}(\mathbf{f})-\mathbf{u}_{N}, w_{N}\right)=0, & \forall w_{N} \in W_{\bar{N}} \\ a\left(\mathbf{u}_{N}^{*}(\mathbf{f})-\mathbf{u}_{N}^{*}, \mathbf{v}_{N}\right)+b\left(\mathbf{v}_{N}, p_{N}^{*}(\mathbf{f})-p_{N}^{*}\right) & \\ \quad=\left(\left(1+\frac{t}{\gamma}\right) \mathbf{u}_{N}(\mathbf{f})-\left(1+\frac{t_{N}}{\gamma}\right) \mathbf{u}_{N}, \mathbf{v}_{N}\right), & \forall \mathbf{v}_{N} \in \mathbf{V}_{N^{\prime}} \\ b\left(\mathbf{u}_{N}^{*}(\mathbf{f})-\mathbf{u}_{N}^{*}, w_{N}\right)=0, & \forall w_{N} \in W_{\bar{N}} .\end{cases}
$$

By the projection $\mathbb{P}_{N}$ in (4.1), one calculates that

$$
\begin{aligned}
\left|\left(\mathbf{f}-\mathbf{f}_{N}, \mathbf{v}_{N}\right)\right| & =\left|\left(\mathbf{f}-\mathbb{P}_{N} \mathbf{f}, \mathbf{v}_{N}\right)+\left(\mathbb{P}_{N} \mathbf{f}-\mathbf{f}_{N}, \mathbf{v}_{N}\right)\right| \\
& \leq C\left\{\left\|\mathbb{P}_{N} \mathbf{f}-\mathbf{f}_{N}\right\|_{0, \Omega}\left\|\mathbf{v}_{N}\right\|_{0, \Omega}+N^{-1}\left\|\mathbf{f}-\mathbb{P}_{N} \mathbf{f}\right\|_{0, \Omega}\left\|\mathbf{v}_{N}\right\|_{1, \Omega}\right\} .
\end{aligned}
$$


On the other hand, in the light of (3.4) and (4.8), we have

$$
\begin{aligned}
\left|\mathbf{u}_{N}-\mathbf{u}_{N}(\mathbf{f})\right|_{1, \Omega}^{2} \leq C & \left\{\left\|\mathbb{P}_{N} \mathbf{f}-\mathbf{f}_{N}\right\|_{0, \Omega}\left\|\mathbf{u}_{N}-\mathbf{u}_{N}(\mathbf{f})\right\|_{0, \Omega}\right. \\
& \left.+N^{-1}\left\|\mathbf{f}-\mathbb{P}_{N} \mathbf{f}\right\|_{0, \Omega}\left\|\mathbf{u}_{N}-\mathbf{u}_{N}(\mathbf{f})\right\|_{1, \Omega}\right\},
\end{aligned}
$$

i.e.,

$$
\left\|\mathbf{u}_{N}-\mathbf{u}_{N}(\mathbf{f})\right\|_{1, \Omega} \leq C\left\{\left\|\mathbb{P}_{N} \mathbf{f}-\mathbf{f}_{N}\right\|_{0, \Omega}+N^{-1}\left\|\mathbf{f}-\mathbb{P}_{N} \mathbf{f}\right\|_{0, \Omega}\right\}
$$

Based on the inf-sup condition (3.3), for $q_{N}=p_{N}-p_{N}(\mathbf{f})$, there holds

$$
\begin{aligned}
\beta\left\|p_{N}-p_{N}(\mathbf{f})\right\|_{L^{2}(\Omega)} & \leq \sup _{\mathbf{v}_{N} \in \mathbf{V}_{N}} \frac{\left(p_{N}-p_{N}(\mathbf{f}), \nabla \cdot \mathbf{v}_{N}\right)}{\left\|\mathbf{v}_{N}\right\|_{1, \Omega}} \\
& =\sup _{\mathbf{v}_{N} \in \mathbf{V}_{N}} \frac{\left(\mathbf{f}-\mathbf{f}_{N}, \mathbf{v}_{N}\right)-a\left(\mathbf{u}_{N}-\mathbf{u}_{N}(\mathbf{f}), \mathbf{v}_{N}\right)}{\left\|\mathbf{v}_{N}\right\|_{1, \Omega}} .
\end{aligned}
$$

Setting $\mathbf{v}_{N}=\mathbf{u}_{N}-\mathbf{u}_{N}(\mathbf{f}) \in \mathbf{V}_{N}$, there holds

$$
C\left\|p_{N}-p_{N}(\mathbf{f})\right\|_{0, \Omega} \leq\left\|\mathbf{f}-\mathbf{f}_{N}\right\|_{0, \Omega}+\left\|\mathbf{u}_{N}-\mathbf{u}_{N}(\mathbf{f})\right\|_{1, \Omega},
$$

where we used that for $\forall \mathbf{v} \in \mathbf{V}$, there holds $\|\nabla \mathbf{v}\|_{0, \Omega}=\|\nabla \times \mathbf{v}\|_{0, \Omega}+\|\nabla \cdot \mathbf{v}\|_{0, \Omega}$. Here the constant $C$ depends on $\Omega$ and $\beta$. This completes the proof of (4.7).

Lemma 4.3. Let $\left(\mathbf{u}_{N}, p_{N}, \mathbf{u}_{N}^{*}, p_{N}^{*}, t_{N}, \mathbf{f}_{N}\right)$ and $\left(\mathbf{u}_{N}(\mathbf{f}), p_{N}(\mathbf{f}), \mathbf{u}_{N}^{*}(\mathbf{f}), p_{N}^{*}(\mathbf{f}), t, \mathbf{f}\right)$ be the solutions of (3.4)) and (4.6)), respectively. Then there holds

$$
\left\|\mathbf{u}_{N}^{*}-\mathbf{u}_{N}^{*}(\mathbf{f})\right\|_{1, \Omega}+\left\|p_{N}^{*}-p_{N}^{*}(\mathbf{f})\right\|_{0, \Omega} \leq C\left\{\left|t-t_{N}\right|+\left\|\mathbf{u}-\mathbf{u}_{N}\right\|_{0, \Omega}\right\},
$$

where $C$ depends on $\Omega$ and $\gamma$.

Proof. By setting $q_{N}=p_{N}^{*}-p_{N}^{*}(\mathbf{f})$ in (3.3), and subtracting the third equality of (4.6) from that of (3.4), we have

$$
\begin{aligned}
& \beta\left\|p_{N}^{*}-p_{N}^{*}(\mathbf{f})\right\|_{L^{2}(\Omega)} \leq \sup _{\mathbf{v}_{N} \in \mathbf{V}_{N}} \frac{\left(p_{N}^{*}-p_{N}^{*}(\mathbf{f}), \nabla \cdot \mathbf{v}_{N}\right)}{\left\|\mathbf{v}_{N}\right\|_{1, \Omega}} \\
= & \sup _{\mathbf{v}_{N} \in \mathbf{V}_{N}} \frac{\left(\left(1+\frac{t}{\gamma}\right) \mathbf{u}-\left(1+\frac{t_{N}}{\gamma}\right) \mathbf{u}_{N}, \mathbf{v}_{N}\right)-a\left(\mathbf{u}_{N}^{*}-\mathbf{u}_{N}^{*}(\mathbf{f}), \mathbf{v}_{N}\right)}{\left\|\mathbf{v}_{N}\right\|_{1, \Omega}} \\
= & \sup _{\mathbf{v}_{N} \in \mathbf{V}_{N}} \frac{\left(\frac{1}{\gamma}\left(t-t_{N}\right) \mathbf{u}+t_{N}\left(\mathbf{u}-\mathbf{u}_{N}\right), \mathbf{v}_{N}\right)-a\left(\mathbf{u}_{N}^{*}-\mathbf{u}_{N}^{*}(\mathbf{f}), \mathbf{v}_{N}\right)}{\left\|\mathbf{v}_{N}\right\|_{1, \Omega}} .
\end{aligned}
$$

Similarly, choosing $\mathbf{v}_{N}=\mathbf{u}_{N}^{*}-\mathbf{u}_{N}^{*}(\mathbf{f}) \in \mathbf{V}_{N}$, one directly calculates that

$$
C\left\|p_{N}^{*}-p_{N}^{*}(\mathbf{f})\right\|_{0, \Omega} \leq\left|t-t_{N}\right|+\left\|\mathbf{u}-\mathbf{u}_{N}\right\|_{0, \Omega}+\left\|\mathbf{u}_{N}^{*}-\mathbf{u}_{N}^{*}(\mathbf{f})\right\|_{1, \Omega} .
$$

Meanwhile, by (4.8), one readily obtains

$$
\left|\mathbf{u}_{N}^{*}-\mathbf{u}_{N}^{*}(\mathbf{f})\right|_{1, \Omega} \leq c\left\{\left|t-t_{N}\right|+\left\|\mathbf{u}-\mathbf{u}_{N}\right\|_{0, \Omega}\right\} .
$$

With the above analyses, the desired (4.9) holds. 
Lemma 4.4. Let $\left(\mathbf{u}_{N}, p_{N}, \mathbf{u}_{N}^{*}, p_{N}^{*}, t_{N}, \mathbf{f}_{N}\right)$ and $\left(\mathbf{u}_{N}(\mathbf{f}), p_{N}(\mathbf{f}), \mathbf{u}_{N}^{*}(\mathbf{f}), p_{N}^{*}(\mathbf{f}), t, \mathbf{f}\right)$ be the solutions of (3.4) and (4.6), respectively. Then there holds

$$
\begin{aligned}
& \left\|\mathbf{f}-\mathbf{f}_{N}\right\|_{0, \Omega} \leq C\left\{\left\|\mathbf{u}^{*}-\mathbf{u}_{N}^{*}(\mathbf{f})\right\|_{0, \Omega}+\left|t-t_{N}\right|\right\}, \\
& \left|t-t_{N}\right| \leq C\left\{\left\|\mathbf{u}^{*}-\mathbf{u}_{N}^{*}(\mathbf{f})\right\|_{1, \Omega}+\left\|\mathbf{u}-\mathbf{u}_{N}(\mathbf{f})\right\|_{0, \Omega}+\left\|p^{*}-p_{N}^{*}(\mathbf{f})\right\|_{0, \Omega}\right\} .
\end{aligned}
$$

Proof. With the Gâteaux derivative of $\mathcal{J}\left(\mathbf{u}_{N}(\mathbf{f}), \mathbf{f}\right)$ following $\mathbf{f}-\mathbf{f}_{N}$, we obtain that

$$
\begin{aligned}
& D \mathcal{J}\left(\mathbf{u}_{N}(\mathbf{f}), \mathbf{f}\right) \cdot\left(\mathbf{f}-\mathbf{f}_{N}\right)=\left(\mathbf{u}_{N}(\mathbf{f})-\mathbf{u}_{d}, D \mathbf{u}_{N}(\mathbf{f}) \cdot\left(\mathbf{f}-\mathbf{f}_{N}\right)\right)+\left(\mathbf{f}, \mathbf{f}-\mathbf{f}_{N}\right) \\
& =\left(\left(1+\frac{t}{\gamma}\right) \mathbf{u}_{N}(\mathbf{f})-\mathbf{u}_{d}, D \mathbf{u}_{N}(\mathbf{f}) \cdot\left(\mathbf{f}-\mathbf{f}_{N}\right)\right)+\left(\mathbf{f}, \mathbf{f}-\mathbf{f}_{N}\right)-\left(\frac{t}{\gamma} \mathbf{u}_{N}(\mathbf{f}), D \mathbf{u}_{N}(\mathbf{f}) \cdot\left(\mathbf{f}-\mathbf{f}_{N}\right)\right) \\
& =\left(\mathbf{u}_{N}^{*}(\mathbf{f})+\mathbf{f}, \mathbf{f}-\mathbf{f}_{N}\right)-\left(\frac{t}{\gamma} \mathbf{u}_{N}(\mathbf{f}), \mathbf{u}_{N}(\mathbf{f})-\mathbf{u}_{N}\right), \\
& D \mathcal{J}\left(\mathbf{u}_{N}\left(\mathbf{f}_{N}\right), \mathbf{f}_{N}\right) \cdot\left(\mathbf{f}-\mathbf{f}_{N}\right)=\left(\mathbf{u}_{N}^{*}+\mathbf{f}_{N}, \mathbf{f}-\mathbf{f}_{N}\right)-\left(\frac{t_{N}}{\gamma} \mathbf{u}_{N}, \mathbf{u}_{N}(\mathbf{f})-\mathbf{u}_{N}\right) .
\end{aligned}
$$

Then by (2.3) and (4.5), there holds

$$
\begin{aligned}
c\left\|\mathbf{f}-\mathbf{f}_{N}\right\|_{0, \Omega}^{2} \leq & \left|D \mathcal{J}\left(\mathbf{u}_{N}\left(\mathbf{f}_{N}\right), \mathbf{f}_{N}\right) \cdot\left(\mathbf{f}-\mathbf{f}_{N}\right)-D \mathcal{J}\left(\mathbf{u}_{N}(\mathbf{f}), \mathbf{f}\right) \cdot\left(\mathbf{f}-\mathbf{f}_{N}\right)\right| \\
= & \left.\left.\mid\left(\mathbf{u}_{N}^{*}+\mathbf{f}_{N}, \mathbf{f}-\mathbf{f}_{N}\right)\right)-\left(\frac{t_{N}}{\gamma} \mathbf{u}_{N}, \mathbf{u}_{N}(\mathbf{f})-\mathbf{u}_{N}\right)-\left(\mathbf{u}_{N}^{*}(\mathbf{f})+\mathbf{f}, \mathbf{f}-\mathbf{f}_{N}\right)\right) \\
& \quad+\left(\frac{t}{\gamma} \mathbf{u}_{N}(\mathbf{f}), \mathbf{u}_{N}(\mathbf{f})-\mathbf{u}_{N}\right) \mid \\
= & \mid\left(\mathbf{u}_{N}^{*}(\mathbf{f})-\mathbf{u}^{*}, \mathbf{f}-\mathbf{f}_{N}\right)+\left(\frac{1}{\gamma}\left(\mathbf{u}_{N}\left(t_{N}-t\right), \mathbf{u}_{N}(\mathbf{f})-\mathbf{u}_{N}\right)\right) \\
& \quad-\frac{t}{\gamma}\left(\mathbf{u}_{N}-\mathbf{u}_{N}(\mathbf{f}), \mathbf{u}_{N}-\mathbf{u}_{N}(\mathbf{f})\right) \mid \\
\leq & \left|\left(\mathbf{u}_{N}^{*}(\mathbf{f})-\mathbf{u}^{*}, \mathbf{f}-\mathbf{f}_{N}\right)+\left(\frac{1}{\gamma}\left(\mathbf{u}_{N}\left(t_{N}-t\right), \mathbf{u}_{N}(\mathbf{f})-\mathbf{u}_{N}\right)\right)\right| \\
\leq & C\left(\frac{1}{\epsilon_{1}}\right)\left\|\mathbf{f}-\mathbf{f}_{N}\right\|_{0, \Omega}^{2}+C\left(\epsilon_{1}\right)\left\|\mathbf{u}^{*}-\mathbf{u}_{N}^{*}(\mathbf{f})\right\|_{0, \Omega} \\
& \quad+C\left(\frac{1}{\epsilon_{2}}\right)\left\|\mathbf{f}-\mathbf{f}_{N}\right\|_{0, \Omega}^{2}+C\left(\epsilon_{2}\right)\left|t-t_{N}\right|,
\end{aligned}
$$

where

$$
\frac{t}{\gamma}\left(\mathbf{u}_{N}-\mathbf{u}_{N}(\mathbf{f}), \mathbf{u}_{N}-\mathbf{u}_{N}(\mathbf{f})\right) \geq 0
$$

and $\epsilon_{1}, \epsilon_{2}$ are some positive constants.

Selecting $\varsigma_{N} \in C_{0}^{\infty}(\Omega)$, such that $\varsigma_{N} \mathbf{u}_{N}(\mathbf{f}) \in \mathbf{V}_{N}$ and

$$
\left\|\sqrt{\varsigma_{N}} \mathbf{u}_{N}(\mathbf{f})\right\|_{0, \Omega} \geq \frac{\gamma}{2}
$$


then with (4.5), we have

$$
\begin{aligned}
& \frac{\gamma^{2}}{4}\left|t-t_{N}\right| \leq\left|\left(t-t_{N}\right)\left(\mathbf{u}_{N}(\mathbf{f}), \varsigma_{N} \mathbf{u}_{N}(\mathbf{f})\right)\right| \\
= & \left|a\left(\mathbf{u}^{*}-\mathbf{u}_{N}^{*}(\mathbf{f}), \varsigma_{N} \mathbf{u}_{N}(\mathbf{f})\right)+b\left(\varsigma_{N} \mathbf{u}_{N}(\mathbf{f}), p^{*}-p_{N}^{*}(\mathbf{f})\right)-\left(t \mathbf{u}-t_{N} \mathbf{u}_{N}(\mathbf{f}), \varsigma_{N} \mathbf{u}_{N}(\mathbf{f})\right)\right| \\
\leq & C\left\{\left\|\mathbf{u}^{*}-\mathbf{u}_{N}^{*}(\mathbf{f})\right\|_{1, \Omega}\left\|\varsigma_{N} \mathbf{u}_{N}(\mathbf{f})\right\|_{1, \Omega}+\left\|p^{*}-p_{N}^{*}(\mathbf{f})\right\|_{0, \Omega}\left\|\varsigma_{N} \mathbf{u}_{N}(\mathbf{f})\right\|_{1, \Omega}\right. \\
& \left.\quad+\left\|\mathbf{u}-\mathbf{u}_{N}(\mathbf{f})\right\|_{0, \Omega}\left\|\varsigma_{N} \mathbf{u}_{N}(\mathbf{f})\right\|_{0, \Omega}\right\} .
\end{aligned}
$$

Combining the above estimates, one readily completes the proof of (4.10).

Lemma 4.5. Let $\left(\mathbf{u}_{N}, p_{N}, \mathbf{u}_{N}^{*}, p_{N}^{*}, t_{N}, \mathbf{f}_{N}\right)$ and $\left(\mathbf{u}_{N}(\mathbf{f}), p_{N}(\mathbf{f}), \mathbf{u}_{N}^{*}(\mathbf{f}), p_{N}^{*}(\mathbf{f}), t, \mathbf{f}\right)$ be the solutions of (3.4) and (4.6), respectively. Then there holds

$$
\begin{gathered}
\left\|\mathbb{P}_{N} \mathbf{f}-\mathbf{f}_{N}\right\|_{0, \Omega} \leq C\left\{\left\|\mathbf{u}_{N}^{*}(\mathbf{f})-\mathbf{u}^{*}\right\|_{0, \Omega}+\left\|\mathbf{u}_{N}^{*}(\mathbf{f})-\mathbf{u}_{N}^{*}\right\|_{0, \Omega}+\left\|\mathbf{u}_{N}(\mathbf{f})-\mathbf{u}\right\|_{0, \Omega}\right. \\
\left.+\left\|\mathbf{u}-\mathbf{u}_{N}\right\|_{0, \Omega}+\left|t-t_{N}\right|\right\} .
\end{gathered}
$$

Proof. Based on the optimality conditions of (3.2) and the auxiliary system, one directly gets that

$$
\begin{aligned}
& \left(t \mathbf{u}-t_{N} \mathbf{u}_{N}, \mathbf{u}_{N}(\mathbf{f})-\mathbf{u}\right)-\left(t \mathbf{u}-t_{N} \mathbf{u}_{N}, \mathbf{u}_{N}(\mathbf{f})-\mathbf{u}_{N}\right) \\
= & t\left(\mathbf{u}, \mathbf{u}_{N}-\mathbf{u}\right)+t_{N}\left(\mathbf{u}_{N}, \mathbf{u}-\mathbf{u}_{N}\right) \leq 0
\end{aligned}
$$

i.e.,

$$
\left(t \mathbf{u}-t_{N} \mathbf{u}_{N}, \mathbf{u}_{N}(\mathbf{f})-\mathbf{u}\right) \leq\left(t \mathbf{u}-t_{N} \mathbf{u}_{N}, \mathbf{u}_{N}(\mathbf{f})-\mathbf{u}_{N}\right)
$$

Then we have

$$
\begin{aligned}
& \left(\mathbb{P}_{N} \mathbf{f}-\mathbf{f}_{N}, \mathbb{P}_{N} \mathbf{f}-\mathbf{f}_{N}\right)=\left(\mathbb{P}_{N} \mathbf{f}-\mathbf{f}_{N}, \mathbf{f}-\mathbf{f}_{N}\right) \\
\leq & \left(\mathbb{P}_{N} \mathbf{f}-\mathbf{f}_{N}, \mathbf{u}_{N}^{*}(\mathbf{f})-\mathbf{u}^{*}\right)-\left(\mathbf{u}-\mathbf{u}_{N}, \mathbf{u}_{N}(\mathbf{f})-\mathbf{u}_{N}\right)-\left(\mathbb{P}_{N} \mathbf{f}-\mathbf{f}, \mathbf{u}_{N}^{*}(\mathbf{f})-\mathbf{u}_{N}^{*}\right) \\
& \quad-\left(t \mathbf{u}-t_{N} \mathbf{u}_{N}, \mathbf{u}_{N}(\mathbf{f})-\mathbf{u}_{N}\right) .
\end{aligned}
$$

And hence, there holds

$$
\begin{aligned}
& \left(\mathbb{P}_{N} \mathbf{f}-\mathbf{f}_{N}, \mathbb{P}_{N} \mathbf{f}-\mathbf{f}_{N}\right) \\
\leq & \left(\mathbb{P}_{N} \mathbf{f}-\mathbf{f}_{N}, \mathbb{P}_{N} \mathbf{f}-\mathbf{f}_{N}\right)+\left(\mathbf{u}_{N}(\mathbf{f})-\mathbf{u}_{N}, \mathbf{u}_{N}(\mathbf{f})-\mathbf{u}_{N}\right) \\
\leq & \left(\mathbb{P}_{N} \mathbf{f}-\mathbf{f}_{N}, \mathbf{u}_{N}^{*}(\mathbf{f})-\mathbf{u}^{*}\right)-\left(\mathbf{u}_{N}(\mathbf{f})-\mathbf{u}, \mathbf{u}_{N}(\mathbf{f})-\mathbf{u}_{N}\right) \\
& \quad-\left(\mathbb{P}_{N} \mathbf{f}-\mathbf{f}, \mathbf{u}_{N}^{*}(\mathbf{f})-\mathbf{u}_{N}^{*}\right)-\left(t \mathbf{u}-t_{N} \mathbf{u}_{N}, \mathbf{u}_{N}(\mathbf{f})-\mathbf{u}_{N}\right) \\
= & \left(\mathbb{P}_{N} \mathbf{f}-\mathbf{f}_{N}, \mathbf{u}_{N}^{*}(\mathbf{f})-\mathbf{u}^{*}\right)-\left(\mathbb{P}_{N} \mathbf{f}-\mathbf{f}, \mathbf{u}_{N}^{*}(\mathbf{f})-\mathbf{u}_{N}^{*}\right) \\
& \quad+\left(\mathbf{u}_{N}(\mathbf{f})-\mathbf{u}_{N}, \mathbf{u}_{N}(\mathbf{f})-\mathbf{u}\right)-\left(t \mathbf{u}-t_{N} \mathbf{u}_{N}, \mathbf{u}_{N}(\mathbf{f})-\mathbf{u}\right) \\
& \quad+\left(\mathbf{u}_{N}(\mathbf{f})-\mathbf{u}, \mathbf{u}_{N}(\mathbf{f})-\mathbf{u}\right)+\left(\mathbf{u}-\mathbf{u}_{N}, \mathbf{u}_{N}(\mathbf{f})-\mathbf{u}\right)-\left(t \mathbf{u}-t_{N} \mathbf{u}_{N}, \mathbf{u}_{N}(\mathbf{f})-\mathbf{u}\right) .
\end{aligned}
$$

One readily obtains that

$$
\begin{aligned}
& c\left\|\mathbb{P}_{N} \mathbf{f}-\mathbf{f}_{N}\right\|_{0, \Omega} \\
\leq & \left\|\mathbf{u}_{N}^{*}(\mathbf{f})-\mathbf{u}^{*}\right\|_{0, \Omega}+\left\|\mathbf{u}_{N}^{*}(\mathbf{f})-\mathbf{u}_{N}^{*}\right\|_{0, \Omega}+\left\|\mathbf{u}_{N}(\mathbf{f})-\mathbf{u}\right\|_{0, \Omega}+\left\|\mathbf{u}-\mathbf{u}_{N}\right\|_{0, \Omega}+\left|t-t_{N}\right| .
\end{aligned}
$$

This is desired error estimates listed in (4.13). 
Now, we combine the above analyses to list our main result and the sketch of the proof.

Theorem 4.1. Assume that the solutions of the optimality conditions have the following regularity properties: $\mathbf{u}, \mathbf{u}^{*} \in \mathbf{H}^{m}(\Omega)$ and $p, p^{*} \in H^{m-1}(\Omega)$. Let $\left(\mathbf{u}, p, \mathbf{u}^{*}, p^{*}, t, \mathbf{f}\right)$ and $\left(\mathbf{u}_{N}, p_{N}, \mathbf{u}_{N}^{*}, p_{N}^{*}, t_{N}, \mathbf{f}_{N}\right)$ be the solutions of (2.11) and (3.4), respectively. Then there holds the following a priori error estimate

$$
\begin{aligned}
& \left\|\mathbf{f}-\mathbf{f}_{N}\right\|_{0, \Omega}+\left\|\mathbf{u}-\mathbf{u}_{N}\right\|_{1, \Omega}+\left\|\mathbf{u}^{*}-\mathbf{u}_{N}^{*}\right\|_{1, \Omega}+\left\|p-p_{N}\right\|_{0, \Omega}+\left\|p^{*}-p_{N}^{*}\right\|_{0, \Omega}+\left|t-t_{N}\right| \\
\leq & c N^{1-m}\left\{\|\mathbf{f}\|_{m-2, \Omega}+\|\mathbf{u}\|_{m, \Omega}+\|p\|_{m-1, \Omega}+\left\|\mathbf{u}^{*}\right\|_{m, \Omega}+\left\|p^{*}\right\|_{m-1, \Omega}\right\} .
\end{aligned}
$$

Proof. By standard techniques for the Stokes equations [6], we readily obtain

$$
\begin{aligned}
& \left\|\mathbf{u}-\mathbf{u}_{N}(\mathbf{f})\right\|_{1, \Omega}+\left\|\mathbf{u}^{*}-\mathbf{u}_{N}^{*}(\mathbf{f})\right\|_{1, \Omega} \leq C N^{1-m}\left\{\|\mathbf{u}\|_{m, \Omega}+\|p\|_{m-1, \Omega}\right\} \\
& \left\|p-p_{N}(\mathbf{f})\right\|_{0, \Omega}+\left\|p^{*}-p_{N}^{*}(\mathbf{f})\right\|_{0, \Omega} \leq C N^{1-m}\left\{\left\|\mathbf{u}^{*}\right\|_{m, \Omega}+\left\|p^{*}\right\|_{m-1, \Omega}\right\} .
\end{aligned}
$$

Combining these estimates in (4.7), (4.9), (4.10), (4.13) with (4.4) and (4.5), one directly deduces that (4.15) holds.

\section{Numerical algorithm and convergence analyses}

The classical Uzawa iteration scheme is an efficient algorithm for solving saddle-point problems (see $[3,7,31,34])$. Also it is well known for solving Stokes equations (see [18,27]). In this section, the Uzawa algorithm and projection scheme are employed to calculate the numerical solutions. We list the corresponding numerical algorithm for (3.4) in Algoritm 5.1 and study the convergence in detail.

\section{Algorithm 5.1.}

Step 1 Set stop criteria $E_{p}, E_{p s}, E_{\mathbf{f}} E_{t}$, and iteration parameters $\rho_{1}, \rho_{2}, \rho_{3}, \rho_{4}$. Select $\lambda \in$ $(0,1)$. Let $s:=0$ and initialize $\hat{p}_{N^{\prime}}^{0}\left(\hat{p}_{N}^{*}\right)^{0}, \hat{\mathbf{f}}_{N}^{0}, t_{N}^{0}$. Iterate over Step 2 to Step 6.

Step 2 Set $m:=0, n:=0, \mathbf{f}_{N}^{s, n}=\hat{\mathbf{f}}_{N}^{s}$.

Step 3 Set $p_{N}^{s, n, m}=\hat{p}_{N}^{n}$ and calculate

$$
\begin{aligned}
& a\left(\mathbf{u}_{N}^{s, n, m}, \mathbf{w}_{N}\right)=-b\left(\mathbf{w}_{N}, p_{N}^{s, n, m}\right)+\left(\mathbf{f}_{N}^{s, n}, \mathbf{w}_{N}\right), \quad \forall \mathbf{w}_{N} \in \mathbf{V}_{N}, \\
& p_{N}^{s, n, m+1}=p_{N}^{s, n, m}-\rho_{1} \nabla \cdot \mathbf{u}_{N}^{s, n, m} .
\end{aligned}
$$

If error of $p_{N}$ satisfies $E_{p}$, stop. Else $\hat{p}_{N}^{n}=p_{N}^{s, n, m+1}, m:=m+1$, and return to Step 3. 
Step 4 With $\mathbf{u}_{N}^{s, n, m}$ from Step 3 and $\left(p_{N}^{*}\right)^{s, n, m}=\left(\hat{p}_{N}^{*}\right)^{n}$. Employ $t_{N}^{s}$ to calculate

$$
\begin{aligned}
& a\left(\left(\mathbf{u}_{N}^{*}\right)^{s, n, m}, \mathbf{w}_{N}\right)=-b\left(\mathbf{w}_{N},\left(p_{N}^{*}\right)^{s, n, m}\right)+\left(\left(1+\frac{t_{N}^{s}}{\gamma}\right) \mathbf{u}_{N}^{s, n, m}-\mathbf{u}_{d}, \mathbf{w}_{N}\right), \quad \forall \mathbf{w}_{N} \in \mathbf{V}_{N}, \\
& \left(p_{N}^{*}\right)^{s, n, m+1}=\left(p_{N}^{*}\right)^{s, n, m}-\rho_{2} \nabla \cdot\left(\mathbf{u}^{*}\right)_{N}^{s, n, m} .
\end{aligned}
$$

If error of $p_{N}^{*}$ satisfies $E_{p s}$, stop. Else $\left(\hat{p}_{N}^{*}\right)^{n}=\left(p_{N}^{*}\right)^{s, n, m+1}, m:=m+1$, return to Step 4 .

Step 5 Set

$$
\mathbf{f}_{N}^{s, n+1}=\mathbf{f}_{N}^{s, n}-\rho_{3}\left(\left(\mathbf{u}_{N}^{*}\right)^{s, n, m}+\mathbf{f}_{N}^{s, n}\right)
$$

If error of $\mathbf{f}_{N}$ satisfies $E_{\mathbf{f}}$, stop. Else $n:=n+1$, and return to Step 3.

Step 6 Calculate

$$
t_{N}^{s+1}=\max \left\{0, t_{N}^{s}+\rho_{4}\left(\left\|\mathbf{u}_{N}^{s, n, m}\right\|_{0, \Omega}-\gamma\right)\right\}
$$

If the error of $t_{N}$ satisfies $E_{t}$, stop. Else $\hat{\mathbf{f}}_{N}^{s+1}=\mathbf{f}_{N}^{s, n+1}$. Then set $s:=s+1$ and return to Step 2.

Hereafter, we give rigorous convergence analyses of the algorithm presented in Algorithm 5.1. For all $v \in H_{0}^{1}(\Omega)$, we recall the Poincaré inequality (see $[2,36]$ )

$$
\|v\|_{0, \Omega} \leq \frac{2}{\sqrt{\pi}}|v|_{1, \Omega}
$$

As we known that one of the key points for solving the Stokes equations (2.1) is to decouple pressure $p$ from velocity $\mathbf{u}$. To investigate the convergence, we introduce the inverse elliptic operator $L$ (more properties please refer to $[28,32]$ ) satisfies

$$
-\Delta \mathscr{Y}=\mathscr{U} \Longleftrightarrow \mathscr{Y}=L \mathscr{U} .
$$

Theorem 5.1. Setting

$$
\rho_{1}=v, \quad \rho_{2}=v, \quad 0<\rho_{3}<1, \quad 0<\rho_{4}<2 \Theta, \quad 0<\lambda<1,
$$

in the above algorithm, we deduce that if the iterations $m \rightarrow+\infty$, there holds

$$
\begin{aligned}
& \left\|\mathbf{u}_{N}^{m}-\mathbf{u}_{N}\right\|_{1, \Omega}+\left\|p_{N}^{m}-p_{N}\right\|_{0, \Omega}+\left\|\mathbf{f}_{N}^{m}-\mathbf{f}_{N}\right\|_{0, \Omega}+\left\|\left(\mathbf{u}_{N}^{*}\right)^{m}-\mathbf{u}_{N}^{*}\right\|_{1, \Omega} \\
& \quad+\left\|\left(p_{N}^{*}\right)^{m}-p_{N}^{*}\right\|_{0, \Omega}+\left|t_{N}^{m}-t_{N}\right| \rightarrow 0 .
\end{aligned}
$$


Proof. For the sake of convenience, we simplify the indexes within the above algorithm. In view of (2.1), there hold

$$
\begin{aligned}
& \mathbf{u}_{N}^{m+1}-\mathbf{u}_{N}=\frac{L\left(\mathbf{f}_{N}^{m}-\mathbf{f}_{N}-\nabla\left(p_{N}^{m}-p_{N}\right)\right)}{v}, \\
& p_{N}^{m+1}-p_{N}=\left(I-\frac{-\rho_{1} \nabla \cdot L \nabla}{v}\right)\left(p_{N}^{m}-p_{N}\right)+\frac{-\rho_{1} \nabla \cdot L}{v}\left(\mathbf{f}_{N}^{m}-\mathbf{f}_{N}\right),
\end{aligned}
$$

where $I$ denotes the identical operator. A particularly effective choice is $\rho_{1}=v$ (see [32]). Since $L$ is self-adjoint and $I-(-\nabla \cdot L \nabla)$ is a strict contraction in $W$ (for more details, please refer to [28]), it is a direct conclusion from the LBB-condition that there holds

$$
\|I-(-\nabla \cdot L \nabla)\|_{\mathscr{L}(W)} \leq 1-\beta^{2},
$$

where $\beta$ is defined in (3.3).

In the light of (5.1), (5.3) and (5.4), we have

$$
\left\|p_{N}^{m}-p_{N}\right\|_{0, \Omega} \leq\left(1-\beta^{2}\right)\left\|p_{N}^{m-1}-p_{N}\right\|_{0, \Omega}+\frac{2}{\sqrt{\pi}}\left\|\mathbf{f}_{N}^{m-1}-\mathbf{f}_{N}\right\|_{0, \Omega}
$$

Meanwhile

$$
\begin{aligned}
& v\left|\mathbf{u}_{N}^{m}-\mathbf{u}_{N}\right|_{1, \Omega}^{2}=a\left(\mathbf{u}_{N}^{m}-\mathbf{u}_{N}, \mathbf{u}_{N}^{m}-\mathbf{u}_{N}\right) \\
= & \left(\mathbf{u}_{N}^{m}-\mathbf{u}_{N},-\nabla\left(p_{N}^{m-1}-p_{N}\right)+\mathbf{f}_{N}^{m-1}-\mathbf{f}_{N}\right) \\
\leq & \left(\left\|p_{N}^{m-1}-p_{N}\right\|_{0, \Omega}+\frac{2}{\sqrt{\pi}}\left\|\mathbf{f}_{N}^{m-1}-\mathbf{f}_{N}\right\|_{0, \Omega}\right)\left|\mathbf{u}_{N}^{m}-\mathbf{u}_{N}\right|_{1, \Omega},
\end{aligned}
$$

i.e.,

$$
\begin{aligned}
& \left\|\mathbf{u}_{N}^{m}-\mathbf{u}_{N}\right\|_{1, \Omega} \leq \sqrt{\frac{4+\pi}{\pi}}\left|\mathbf{u}_{N}^{m}-\mathbf{u}_{N}\right|_{1, \Omega} \\
\leq & \frac{1}{v} \sqrt{\frac{4+\pi}{\pi}}\left(\left\|p_{N}^{m-1}-p_{N}\right\|_{0, \Omega}+\frac{2}{\sqrt{\pi}}\left\|\mathbf{f}_{N}^{m-1}-\mathbf{f}_{N}\right\|_{0, \Omega}\right) .
\end{aligned}
$$

Similarly, with the co-state equations and $\rho_{2}=v$, there hold

$$
\begin{aligned}
& \left(\mathbf{u}_{N}^{*}\right)^{m+1}-\mathbf{u}_{N}^{*}=\frac{L\left(\left(1+\frac{t_{N}^{m}}{\gamma}\right) \mathbf{u}_{N}^{m}-\left(1+\frac{t_{N}}{\gamma}\right) \mathbf{u}_{N}-\nabla\left(\left(p_{N}^{*}\right)^{m}-p_{N}^{*}\right)\right)}{v}, \\
& \left(p_{N}^{*}\right)^{m+1}-p_{N}^{*}=(I-(-\nabla \cdot L \nabla))\left(\left(p_{N}^{*}\right)^{m}-p_{N}^{*}\right)-\nabla \cdot L\left(\left(1+\frac{t_{N}^{m}}{\gamma}\right) \mathbf{u}_{N}^{m}-\left(1+\frac{t_{N}}{\gamma}\right) \mathbf{u}_{N}\right) .
\end{aligned}
$$

One readily knows that

$$
\begin{aligned}
& \left\|\left(p_{N}^{*}\right)^{m}-p_{N}^{*}\right\|_{0, \Omega} \leq\left(1-c_{N}^{2}\right)\left\|\left(p_{N}^{*}\right)^{m-1}-p_{N}^{*}\right\|_{0, \Omega}+\frac{2}{\sqrt{\pi}}\left\|\left(1+\frac{t_{N}^{m}}{\gamma}\right) \mathbf{u}_{N}^{m}-\left(1+\frac{t_{N}}{\gamma}\right) \mathbf{u}_{N}\right\|_{0, \Omega} \\
\leq & \left(1-\beta^{2}\right)\left\|\left(p_{N}^{*}\right)^{m-1}-p_{N}^{*}\right\|_{0, \Omega}+\frac{2}{\sqrt{\pi}}\left\|\mathbf{u}_{N}^{m}-\mathbf{u}_{N}\right\|_{0, \Omega}+\frac{2 C}{\gamma \sqrt{\pi}}\left(\left\|t_{N}^{m}-t_{N}\right\|_{0, \Omega}+\left\|\mathbf{u}_{N}^{m}-\mathbf{u}_{N}\right\|_{0, \Omega}\right) \\
= & \left(1-\beta^{2}\right)\left\|\left(p_{N}^{*}\right)^{m-1}-p_{N}^{*}\right\|_{0, \Omega}+\frac{2}{\sqrt{\pi}}\left(1+\frac{C}{\gamma}\right)\left\|\mathbf{u}_{N}^{m}-\mathbf{u}_{N}\right\|_{0, \Omega}+\frac{2 C}{\gamma \sqrt{\pi}}\left\|t_{N}^{m}-t_{N}\right\|_{0, \Omega}
\end{aligned}
$$


It is clear that

$$
\begin{aligned}
& v\left|\left(\mathbf{u}_{N}^{*}\right)^{m}-\mathbf{u}_{N}^{*}\right|_{1, \Omega}^{2}=a\left(\left(\mathbf{u}_{N}^{*}\right)^{m}-\mathbf{u}_{N}^{*},\left(\mathbf{u}_{N}^{*}\right)^{m}-\mathbf{u}_{N}^{*}\right) \\
= & \left(\left(\mathbf{u}_{N}^{*}\right)^{m}-\mathbf{u}_{N}^{*},-\nabla\left(\left(p_{N}^{*}\right)^{m-1}-p_{N}^{*}\right)+\left(1+\frac{t_{N}^{m}}{\gamma}\right) \mathbf{u}_{N}^{m}-\left(1+\frac{t_{N}}{\gamma}\right) \mathbf{u}_{N}\right) \\
= & \left(\nabla \cdot\left(\left(\mathbf{u}_{N}^{*}\right)^{m}-\mathbf{u}_{N}^{*}\right),\left(p_{N}^{*}\right)^{m-1}-p_{N}^{*}\right)+\left(\left(\mathbf{u}_{N}^{*}\right)^{m}-\mathbf{u}_{N}^{*},\left(1+\frac{t_{N}^{m}}{\gamma}\right) \mathbf{u}_{N}^{m}-\left(1+\frac{t_{N}}{\gamma}\right) \mathbf{u}_{N}\right) \\
\leq & \left(\left\|\left(p_{N}^{*}\right)^{m-1}-p_{N}^{*}\right\|_{0, \Omega}+\frac{2}{\sqrt{\pi}}\left\|\left(1+\frac{t_{N}^{m}}{\gamma}\right) \mathbf{u}_{N}^{m}-\left(1+\frac{t_{N}}{\gamma}\right) \mathbf{u}_{N}\right\|_{0, \Omega}\right)\left(\mathbf{u}_{N}^{*}\right)^{m}-\left.\mathbf{u}_{N}^{*}\right|_{1, \Omega},
\end{aligned}
$$

i.e.,

$$
\begin{aligned}
& v\left\|\left(\mathbf{u}_{N}^{*}\right)^{m}-\mathbf{u}_{N}^{*}\right\|_{1, \Omega} \\
\leq & \sqrt{\frac{4+\pi}{\pi}}\left(\left\|\left(p_{N}^{*}\right)^{m-1}-p_{N}^{*}\right\|_{0, \Omega}+\frac{2}{\sqrt{\pi}}\left\|\left(1+\frac{t_{N}^{m}}{\gamma}\right) \mathbf{u}_{N}^{m}-\left(1+\frac{t_{N}}{\gamma}\right) \mathbf{u}_{N}\right\|_{0, \Omega}\right) \\
\leq & \sqrt{\frac{4+\pi}{\pi}}\left(\left\|\left(p_{N}^{*}\right)^{m-1}-p_{N}^{*}\right\|_{0, \Omega}+\frac{2}{\sqrt{\pi}}\left(1+\frac{C}{\gamma}\right)\left\|\mathbf{u}_{N}^{m}-\mathbf{u}_{N}\right\|_{0, \Omega}+\frac{2 C}{\gamma \sqrt{\pi}}\left\|t_{N}^{m}-t_{N}\right\|_{0, \Omega}\right) .
\end{aligned}
$$

We are at the point to investigate the convergence of $\mathbf{f}_{N}^{m}$.

$$
\begin{aligned}
\left\|\mathbf{f}_{N}^{m}-\mathbf{f}_{N}\right\|_{0, \Omega} & =\left\|\mathbf{f}_{N}^{m-1}-\mathbf{f}_{N}-\rho_{3}\left(\left(\mathbf{u}_{N}^{*}\right)^{m-1}-\mathbf{u}_{N}^{*}+\mathbf{f}_{N}^{m-1}-\mathbf{f}_{N}\right)\right\|_{0, \Omega} \\
& \leq\left(1-\rho_{3}\right)\left\|\mathbf{f}_{N}^{m-1}-\mathbf{f}_{N}\right\|_{0, \Omega}+\rho_{3}\left\|\left(\mathbf{u}_{N}^{*}\right)^{m-1}-\mathbf{u}_{N}^{*}\right\|_{0, \Omega}
\end{aligned}
$$

Next, we turn to analyze the convergence of $t_{N}^{m}$. The function $F(\cdot)$ in (2.10) is Lipschitz, i.e., for $\forall \mathbf{v}, \mathbf{w} \in \mathbf{V}$, there exists a constant $C_{0}$, such that

$$
\|F(\mathbf{v})-F(\mathbf{w})\|_{0, \Omega} \leq C_{0}\|\mathbf{v}-\mathbf{w}\|_{0, \Omega} .
$$

And the Lipschitz constant of $F(\mathbf{u})$ is $C_{0}=\sqrt{|\Omega|}$, since

$$
\begin{gathered}
\frac{\|F(\mathbf{v})-F(\mathbf{w})\|_{0, \Omega}^{2}}{|\Omega|}=\left(\|\mathbf{v}\|_{0, \Omega}-\|\mathbf{w}\|_{0, \Omega}\right)^{2} \\
\leq\left(\|\mathbf{v}\|_{0, \Omega}^{2}+\|\mathbf{w}\|_{0, \Omega}^{2}-2 \int_{\Omega} \mathbf{v} \cdot \mathbf{w}\right)=\|\mathbf{v}-\mathbf{w}\|_{0, \Omega}^{2} .
\end{gathered}
$$

Note that the pair $(\mathbf{u}, t)$ is the optimal solution of the continuous systems, then as in [33], for $\forall \mathbf{v} \in \mathbf{V}$, there holds

$$
\mathcal{J}(\mathbf{u}, \mathbf{f})+\frac{1}{|\Omega|}(t, F(\mathbf{u})) \leq \mathcal{J}(\mathbf{v}, \mathbf{f})+\frac{1}{|\Omega|}(t, F(\mathbf{v})) .
$$

By the constraint $\|\mathbf{u}\|_{0, \Omega} \leq \gamma$, we declare that (5.11) is equivalent to

$$
\left(\mathcal{J}^{\prime}(\mathbf{u}, \mathbf{f}), \mathbf{v}-\mathbf{u}\right)+\frac{1}{|\Omega|}(t, F(\mathbf{v})-F(\mathbf{u})) \geq 0 .
$$


Similarly, for the pairs $\left(\mathbf{u}_{N}, t_{N}\right)$ and $\left(\mathbf{u}_{N}^{m}, t_{N}^{m}\right)$, there hold

$$
\begin{aligned}
& \left(\mathcal{J}^{\prime}\left(\mathbf{u}_{N}, \mathbf{f}_{N}\right), \mathbf{v}-\mathbf{u}_{N}\right)+\frac{1}{|\Omega|}\left(t_{N}, F(\mathbf{v})-F\left(\mathbf{u}_{N}\right)\right) \geq 0, \\
& \left(\mathcal{J}^{\prime}\left(\mathbf{u}_{N}^{m}, \mathbf{f}_{N}^{m}\right), \mathbf{v}-\mathbf{u}_{N}^{m}\right)+\frac{1}{|\Omega|}\left(t_{N}^{m}, F(\mathbf{v})-F\left(\mathbf{u}_{N}^{m}\right)\right) \geq 0 .
\end{aligned}
$$

Taking $\mathbf{v}=\mathbf{u}_{N}^{m}\left(\right.$ resp. $\left.\mathbf{v}=\mathbf{u}_{N}\right)$ in $(5.12 a)($ resp. $(5.12 b))$, we have

$$
\left(\mathcal{J}^{\prime}\left(\mathbf{u}_{N}^{m}\right)-\mathcal{J}^{\prime}\left(\mathbf{u}_{N}\right), \mathbf{u}_{N}^{m}-\mathbf{u}_{N}\right)+\frac{1}{|\Omega|}\left(t_{N}^{m}-t_{N}, F\left(\mathbf{u}_{N}^{m}\right)-F\left(\mathbf{u}_{N}\right)\right) \leq 0 .
$$

With the iterative scheme, we obtain

$$
\begin{aligned}
& \left|t_{N}^{m+1}-t_{N}\right| \leq\left|t_{N}^{m}-t_{N}+\rho_{4}\left(F\left(\mathbf{u}_{N}^{m}\right)-F\left(\mathbf{u}_{N}\right)\right)\right| \\
& \left\|t_{N}^{m+1}-t_{N}\right\|_{0, \Omega}^{2} \leq\left\|t_{N}^{m}-t_{N}+\rho_{4}\left(F\left(\mathbf{u}_{N}^{m}\right)-F\left(\mathbf{u}_{N}\right)\right)\right\|_{0, \Omega}^{2} \\
& =\left\|t_{N}^{m}-t_{N}\right\|_{0, \Omega}^{2}+\rho_{4}^{2}\left\|F\left(\mathbf{u}_{N}^{m}\right)-F\left(\mathbf{u}_{N}\right)\right\|_{0, \Omega}^{2}+2 \rho_{4}\left(t_{N}^{m}-t_{N}, F\left(\mathbf{u}_{N}^{m}\right)-F\left(\mathbf{u}_{N}\right)\right) \\
& \leq\left\|t_{N}^{m}-t_{N}\right\|_{0, \Omega}^{2}+|\Omega| \rho_{4}^{2}\left\|\mathbf{u}_{N}^{m}-\mathbf{u}_{N}\right\|_{0, \Omega}^{2}-2|\Omega|^{2} \rho_{4}\left(\mathcal{J}^{\prime}\left(\mathbf{u}_{N}^{m}\right)-\mathcal{J}^{\prime}\left(\mathbf{u}_{N}\right), \mathbf{u}_{N}^{m}-\mathbf{u}_{N}\right) \\
& \leq\left\|t_{N}^{m}-t_{N}\right\|_{0, \Omega}^{2}+|\Omega| \rho_{4}^{2}\left\|\mathbf{u}_{N}^{m}-\mathbf{u}_{N}\right\|_{0, \Omega}^{2}-2|\Omega|^{2} \Theta \rho_{4}\left\|\mathbf{u}_{N}^{m}-\mathbf{u}_{N}\right\|_{0, \Omega}^{2} \\
& =\left\|t_{N}^{m}-t_{N}\right\|_{0, \Omega}^{2}+|\Omega| \rho_{4}\left(\rho_{4}-2|\Omega| \Theta\right)\left\|\mathbf{u}_{N}^{m}-\mathbf{u}_{N}\right\|_{0, \Omega}^{2} .
\end{aligned}
$$

Hence, if $\rho_{4}$ satisfies

$$
0<\rho_{4}<2 \Theta,
$$

then we readily get that $\left\{\left\|t_{N}^{m+1}-t_{N}\right\|_{0, \Omega}\right\}$ is strictly monotonically decreasing with a lower bound (i.e., zero). And there holds

$$
\left\|t_{N}^{m+1}-t_{N}\right\|_{0, \Omega}-\left\|t_{N}^{m}-t_{N}\right\|_{0, \Omega} \rightarrow 0, \quad m \rightarrow+\infty .
$$

Since $\left\{t_{N}^{m}\right\}$ is bounded, there exists a subsequence $\left\{t_{N}^{\tilde{m}}\right\}$ such that

$$
t_{N}^{\tilde{m}} \rightarrow \tilde{t}_{N}
$$

The corresponding pair $\left(\mathbf{u}_{N}^{\tilde{m}}, t_{N}^{\tilde{m}}\right)$, which lies within the subsequence, satisfies (3.4). In view of $\mathbf{u}_{N}^{\tilde{m}} \rightarrow \mathbf{u}_{N}$, the uniqueness and the above analyses could be repeated for any subsequence of $\left\{t_{N}^{m}\right\}$, then there holds $t_{N}^{m} \rightarrow t_{N}$ [12]. Then

$$
\left\|\mathbf{u}_{N}^{m}-\mathbf{u}_{N}\right\|_{0, \Omega} \rightarrow 0, \quad m \rightarrow+\infty .
$$

Following (5.5)-(5.9), we deduce that if $m \rightarrow+\infty$, there hold

$$
\left\|\left(p_{N}^{*}\right)^{m}-p_{N}^{*}\right\|_{0, \Omega} \rightarrow 0, \quad\left\|\left(\mathbf{u}_{N}^{*}\right)^{m}-\mathbf{u}_{N}^{*}\right\|_{1, \Omega} \rightarrow 0, \quad\left\|\mathbf{f}_{N}^{m}-\mathbf{f}_{N}\right\|_{0, \Omega} \rightarrow 0, \quad\left\|p_{N}^{m}-p_{N}\right\|_{0, \Omega} \rightarrow 0 .
$$

Combining with the above results, we complete the proof of (5.2). 


\section{Numerical experiments}

In this section, for simplicity, we set $\lambda=0.5$. Let $\Omega=(-1,1)^{2}$ and $\gamma=1$ within $K$. And we focus on the model problem as follows

$$
\mathcal{J}(\mathbf{u}, \mathbf{f})=\min \left\{\frac{1}{2} \int_{\Omega}\left|\mathbf{u}-\mathbf{u}_{d}\right|^{2} d x+\frac{1}{2} \int_{\Omega}|\mathbf{f}|^{2} d x\right\}
$$

subjected to

$$
\begin{cases}-v \Delta \mathbf{u}+\nabla p=\mathbf{s}+\mathbf{f} & \text { in } \Omega, \\ \nabla \cdot \mathbf{u}=0 & \text { in } \Omega, \\ \left.\mathbf{u}\right|_{\partial \Omega}=\mathbf{0}, & \\ \|\mathbf{u}\|_{0, \Omega} \leq 1, & \end{cases}
$$

where

$$
v=0.1, \quad \mathbf{s}=-0.1 \Delta \mathbf{u}+\nabla p-\mathbf{f}, \quad \mathbf{u}_{d}=0.1 \Delta \mathbf{u}^{*}-\nabla p^{*}+\left(1+\frac{t}{\gamma}\right) \mathbf{u} .
$$

Example 6.1. In this example, we choose exact solutions of (6.1)-(6.2) as

$$
\begin{array}{lll}
u_{1}=\frac{125}{8 C^{0}}\left(x_{1}^{2}-1\right)^{2} x_{2}\left(x_{2}^{2}-1\right), & u_{2}=-\frac{125}{8 C^{0}} x_{1}\left(x_{1}^{2}-1\right)\left(x_{2}^{2}-1\right)^{2}, & p=\sin \left(\pi x_{1}\right) \sin \left(\pi x_{2}\right), \\
f_{1}=-\frac{4 C^{0}}{5} u_{1}, & f_{2}=-\frac{4 C^{0}}{5} u_{2}, & u_{1}^{*}=\frac{4 C^{0}}{5} u_{1}, \\
u_{2}^{*}=\frac{4 C^{0}}{5} u_{2}, & p^{*}=-\frac{4}{5} p, & t=C^{0}-1,
\end{array}
$$

where

$$
C^{0} \cong 7.77615
$$

such that $\|\mathbf{u}\|_{0, \Omega}=\gamma(\gamma=1)$. The numerical results are listed in the following table.

Table 1: Numerical results with $\rho_{i}=0.1(i=1,2,3,4)$.

\begin{tabular}{||c|c|c|c|c|c||}
\hline$N$ & 6 & 8 & 12 & 16 & 24 \\
\hline$\left\|\mathbf{f}-\mathbf{f}_{N}\right\|_{0, \Omega}$ & $2.98715 \mathrm{e}-2$ & $5.74329 \mathrm{e}-5$ & $1.42235 \mathrm{e}-06$ & $1.41023 \mathrm{e}-09$ & $1.40593 \mathrm{e}-11$ \\
\hline$\left\|\mathbf{u}-\mathbf{u}_{N}\right\|_{1, \Omega}$ & $8.56804 \mathrm{e}-3$ & $1.16318 \mathrm{e}-4$ & $1.11867 \mathrm{e}-06$ & $1.08505 \mathrm{e}-09$ & $1.07804 \mathrm{e}-11$ \\
\hline$\left\|\mathbf{u}^{*}-\mathbf{u}_{N}^{*}\right\|_{1, \Omega}$ & $2.99237 \mathrm{e}-2$ & $5.71137 \mathrm{e}-5$ & $1.28763 \mathrm{e}-06$ & $1.27432 \mathrm{e}-09$ & $1.26963 \mathrm{e}-11$ \\
\hline$\left\|p-p_{N}\right\|_{0, \Omega}$ & $3.05019 \mathrm{e}-1$ & $7.33715 \mathrm{e}-4$ & $2.65861 \mathrm{e}-07$ & $1.63371 \mathrm{e}-10$ & $1.20453 \mathrm{e}-12$ \\
\hline$\left\|p^{*}-p_{N}^{*}\right\|_{0, \Omega}$ & $2.75959 \mathrm{e}-1$ & $5.74238 \mathrm{e}-4$ & $2.32673 \mathrm{e}-07$ & $5.98925 \mathrm{e}-10$ & $2.93066 \mathrm{e}-12$ \\
\hline$\left|t-t_{N}\right|$ & $9.97109 \mathrm{e}-3$ & $9.97505 \mathrm{e}-5$ & $9.97362 \mathrm{e}-07$ & $9.96703 \mathrm{e}-09$ & $9.95959 \mathrm{e}-11$ \\
\hline
\end{tabular}


Example 6.2. In this example, we choose trigonometric functions to design the exact solutions of the optimal control system (6.1)-(6.2). The exact solutions read

$$
\begin{array}{lll}
u_{1}=\cos ^{2}\left(\frac{\pi}{2} x_{1}\right) \sin \left(\pi x_{2}\right), & u_{2}=-\sin \left(\pi x_{1}\right) \cos ^{2}\left(\frac{\pi}{2} x_{2}\right), & p=\sin \left(\pi x_{1}\right) \sin \left(\pi x_{2}\right), \\
f_{1}=-u_{1}, & f_{2}=-u_{2}, & u_{1}^{*}=u_{1}, \\
u_{2}^{*}=u_{2}, & p^{*}=-p, & t=0.22474,
\end{array}
$$

where $\|\mathbf{u}\|_{0, \Omega}=1.22474$. And numerical results are listed in the following table.

Table 2: Numerical results with $\rho_{1}=\rho_{2}=0.1$ and $\rho_{3}=\rho_{4}=0.2$.

\begin{tabular}{||c|c|c|c|c|c|c||}
\hline$N$ & 8 & 10 & 12 & 16 & 24 & 32 \\
\hline$\left\|\mathbf{f}-\mathbf{f}_{N}\right\|_{0, \Omega}$ & $3.07453 \mathrm{e}-2$ & $2.92233 \mathrm{e}-3$ & $3.02496 \mathrm{e}-6$ & $5.84244 \mathrm{e}-9$ & $5.84663 \mathrm{e}-11$ & $5.99651 \mathrm{e}-14$ \\
\hline$\left\|\mathbf{u}-\mathbf{u}_{N}\right\|_{1, \Omega}$ & $2.62449 \mathrm{e}-2$ & $2.46419 \mathrm{e}-3$ & $2.54729 \mathrm{e}-6$ & $4.68015 \mathrm{e}-9$ & $4.68304 \mathrm{e}-11$ & $4.88767 \mathrm{e}-14$ \\
\hline$\left\|\mathbf{u}^{*}-\mathbf{u}_{N}^{*}\right\|_{1, \Omega}$ & $1.66547 \mathrm{e}-2$ & $1.71949 \mathrm{e}-3$ & $1.79395 \mathrm{e}-6$ & $3.30015 \mathrm{e}-9$ & $3.29811 \mathrm{e}-11$ & $3.35228 \mathrm{e}-14$ \\
\hline$\left\|p-p_{N}\right\|_{0, \Omega}$ & $2.58952 \mathrm{e}-3$ & $2.30353 \mathrm{e}-4$ & $2.35886 \mathrm{e}-7$ & $4.32163 \mathrm{e}-10$ & $4.19505 \mathrm{e}-12$ & $4.82955 \mathrm{e}-15$ \\
\hline$\left\|p^{*}-p_{N}^{*}\right\|_{0, \Omega}$ & $3.51652 \mathrm{e}-3$ & $2.32745 \mathrm{e}-4$ & $2.15382 \mathrm{e}-7$ & $3.50804 \mathrm{e}-10$ & $2.93379 \mathrm{e}-12$ & $3.21185 \mathrm{e}-15$ \\
\hline$\left|t-t_{N}\right|$ & $4.69989 \mathrm{e}-2$ & $4.75637 \mathrm{e}-3$ & $4.95443 \mathrm{e}-6$ & $9.58506 \mathrm{e}-9$ & $9.58975 \mathrm{e}-11$ & $9.97813 \mathrm{e}-14$ \\
\hline$\|\mathbf{u}\|_{0, \Omega}-\gamma$ & $2.61056 \mathrm{e}-2$ & $2.45895 \mathrm{e}-3$ & $2.54333 \mathrm{e}-6$ & $4.67534 \mathrm{e}-9$ & $4.67977 \mathrm{e}-11$ & $4.90719 \mathrm{e}-14$ \\
\hline
\end{tabular}

Here we set an additional $\mathbf{s}$ in the right hand side term to simplify the true solution. Above two tables show the error results of spectral approximations for state, adjointstate, control and Lagrangian multiplier variables. We can see that the numerical approximations approach the true solutions as the approximation degrees grow, and the errors decrease rapidly with the given smooth right hand side terms, which are composed of either polynomials or trigonometric functions. Also, the numerical tests show that the high accuracy of spectral methods for this constrained optimal control problem. And during the ongoing work, we are focusing on how to accelerate the iterations and reduce the computational costs.

\section{Conclusions}

In this paper, we study the convergence analyses and a priori error estimates of LegendreGalerkin spectral method for the constrained optimal control problems governed by Stokes equations. The key difference between our work and the topic in [9] is the constraint set, which includes the constraint type and the constrained variables. And with several real parameters $\lambda$, we choose appropriate pairs of discretization spaces for the velocity and pressure to satisfy the LBB-condition. With the help of the first-order equivalent optimal conditions, we depict that the numerical approximations arrive at optimal order in $H^{1}$ - and $L^{2}$ - norms in the a priori error estimates. Specially, $\beta$ in (3.3) is independent of $N$ for $\bar{N}=\lambda N$ and $N \geq 2 /(1-\lambda)$. Another highlight is that our proposed algorithm, which combining with the Uzawa algorithm and a projection scheme, gives 
an effective numerical formula for the saddle-point system of convex programming problems. With rigourous analyses, we give the convergence of our proposed algorithm. The same techniques can be used to study error estimates and convergence analyses for other constraint optimal control cases.

\section{Acknowledgements}

This work is supported by NSFC grants (Nos. 11926355, and 11701253), NSF of Henan Province (No. 15A110024), NSF of Shandong Province (Nos. ZR2019YQ05, 2019KJI003, and 2017GSF216001) and China Postdoctoral Science Foundation (Nos. 2017T100030, and 2017M610751). The third author thanks Prof. Jie Shen (Department of Mathematics, Purdue University) for helpful suggestions and discussions.

\section{References}

[1] F. Abergel AND R. TEMAM, On some optimal control problems in fluid mechanics, Theor. Comput. Fluid Dyn., 6 (1990), pp. 303-325.

[2] R. A. AdAms And J. J. Fournier, Sobolev Spaces, Academic Press, 2003.

[3] K. ARrow, L. Hurwicz AND H. UzAWA, Studies in Nonlinear Programming, Stanford University Press, Stanford, CA, 1958.

[4] F. Auteri, J. L. Guermond And N. PAROLIni, Role of the LBB condition in weak spectral projection methods, J. Comput. Phys., 174 (2001), pp. 405-420.

[5] C. BERNARDI AND Y. MADAY, Uniform inf-sup conditions for the spectral discretization of the stokes problem, Math. Mod. Meth. Appl. S., 9 (2011), pp. 395-414.

[6] C. Canuto, M. Y. Hussaini, A. Quarteroni And T. A. ZAnG, Spectral Methods in Fluid Dynamics, Springer-Verlag, 1988.

[7] Z. H. CAO, Fast Uzawa algorithm for generalized saddle point problems, Appl. Numer. Math., 46 (2003), pp. 157-171.

[8] E. CASAS, Error estimates for the numerical approximation of semilinear elliptic control problems with finitely many state constraints, ESAIM Control Optim. Calc. Var., 8 (2002), pp. 345-374.

[9] Y. P. Chen, F. L. HuAnG, N. Y. YI AND W. B. LIU, A Legendre-Galerkin spectral method for optimal control problems governed by Stokes equations, SIAM J. Numer. Anal., 49 (2011), pp. 1625-1648.

[10] Y. P. CHEN, Y. Q. HuANG AND N. Y. YI, A posteriori error estimates of spectral method for optimal control problems governed by parabolic equations, Sci. China Ser. A, 51 (2008), pp. 13761390.

[11] Y. P. CHEN, N. Y. YI AND W. B. LIU, A Legendre-Galerkin spectral method for optimal control problems governed by elliptic equations, SIAM J. Numer. Anal., 46 (2008), pp. 2254-2275.

[12] P. G. CiARlet, Introduction to Numerical Linear Algebra and Optimisation, Cambridge University Press, 1989.

[13] F. H. ClARKe, Optimization and Nonsmooth Analysis, SIAM, 1990.

[14] P. Constantin, Navier-Stokes Equations, University of Chicago Press, 1988.

[15] J. C. DE LOS REYES AND R. GRIESSE, State-constrained optimal control of the three-dimensional stationary Navier-Stokes equations, J. Math. Anal. Appl., 343 (2008), pp. 257-272. 
[16] M. DeSAI AND K. ITO, Optimal controls of Navier-Stokes equations, SIAM J. Control Optim., 32 (1994), pp. 1428-1446.

[17] M. FORTIN AND R. GLOWINSKI, Augmented Lagrangian Methods: Applications to the Numerical Solution of Boundary-Value Problems, Elsevier, 2000.

[18] H. C. ElmAN AND G. H. GOLUB, Inexact and preconditioned Uzawa algorithms for saddle point problems, SIAM J. Numer. Anal., 31 (1994), pp. 1645-1661.

[19] J. D. YANG, K. ZHANG, AND H. M. SONG ET AL., An alternating direction method of multipliers for optimal control problems constrained with elliptic equations, Adv. Appl. Math. Mech., 12 (2020), pp. 336-361.

[20] M. D. GunZBurger, L. S. Hou AND T. P. SvobodnY, Boundary velocity control of incompressible flow with an application to viscous drag reduction, SIAM J. Control Optim., 30 (1992), pp. 167-181.

[21] M. HinZE, Optimal and Instantaneous Control of the Instaionary Navier-Stokes Equations, Habilitation thesis, Berlin, 2002.

[22] L. S. HOU AND S. S. RAVINDRAN, A penalized Neumann control approach for solving an optimal Dirichlet control problem for the Navier-Stokes equations, SIAM J. Control Optim., 36 (1998), pp. 1795-1814.

[23] R. LI, W. B. LIU, H. P. MA AND T. TANG, Adaptive finite element approximation for distributed elliptic optimal control problems, SIAM J. Control Optim., 41 (2002), pp. 1321-1349.

[24] J. L. LiONS, Optimal Control of System Governed by Partial Differential Equations, Springer, Berlin, 1971.

[25] W. B. LIU, W. GONG AND N. N. YAN, A new finite element approximation of a state-constrained optimal control problems, J. Comput. Math., 27 (2009), pp. 97-114.

[26] W. B. LIU AND N. N. YAN, A posteriori error estimates for control problems governed by Stokes equations, SIAM J. Numer. Anal., 40 (2002), pp. 1850-1869.

[27] Y. Maday, D. Meiron, A. T. Patera AND E. M. RØnQuist, Analysis of iterative methods for the steady and unsteady Stokes problem: Application to spectral element discretizations, SIAM J. Sci. Comput., 14 (1993), pp. 310-337.

[28] M. MARION AND T. TEMAM, Navier-Stokes Equations: Theory and Approximation, in: Handbook of Numerical Analysis, North-Holland, Amsterdam, (1998), pp. 503-688.

[29] H. F. NIU AND D. P. YANG, Finite element analysis of optimal control problems governed by Stokes equations with $L^{2}$-norm state- constraints, J. Comput. Math., 29 (2011), pp. 589-604.

[30] H. F. NIU, D. P. YANG AND L. YUAN, Finite element approximation and analysis of optimal control problems of Stokes equations, Int. J. Numer. Meth. Fl., 69 (2012), pp. 534-549.

[31] W. QUECK, The convergence factor of preconditioned algorithms of the Arrow-Hurwicz type, SIAM J. Numer. Anal., 26 (1989), pp. 1016-1030.

[32] J. Shen, T. TANG AND L. L. WANG, Spectral Methods: Algorithms, Analysis and Applications, Springer, Heidelberg, 2011.

[33] R. TRemolieres, J. L. LiONS AND R. GlOWINSKI, Numerical Analysis of Variational Inequalities, Elsevier, 2011.

[34] H. UzAwA, Iterative Methods for Concave Programming, in K. J. Arrow, L. Hurwicz, H. Uzawa (eds.), Studies in Linear \& Nonlinear Programming, pages 154-165, Stanford University Press, Stanford, 1958.

[35] H. B. GUAN, Y. YANG AND H. Q. ZHU, The convergence and superconvergence of a MFEM for elliptic optimal control problems, Adv. Appl. Math. Mech., 12 (2020), pp. 527-544.

[36] J. W. ZHOU AND D. P. YANG, Spectral mixed Galerkin method for state constrained optimal control problem governed by the first bi-harmonic equation, Int. J. Comput. Math., 88 (2011), pp. 
2988-3011.

[37] J. W. ZHOU AND D. P. YANG, Legendre-Galerkin spectral methods for optimal control problems with integral constraint for state in one dimension, Comput. Optim. Appl., 61 (2015), pp. 135158.

[38] Z. J. ZHOU AND C. ZHANG, Time-stepping discontinuous Galerkin approximation of optimal control problem governed by time fractional diffusion equation, Numer. Algor., 79 (2018), pp. 437455.

[39] Z. J. ZHOU AND W. GONG, Finite element approximation of optimal control problems governed by time fractional diffusion equation, Comput. Math. Appl., 71 (2016), pp. 301-318. 